Article

\title{
Quantum Discord and Information Deficit in Spin Chains
}

\section{Norma Canosa, Leonardo Ciliberti and Raúl Rossignoli *}

Departamento de Física-IFLP, Universidad Nacional de La Plata, C.C.67, La Plata 1900, Argentina;

E-Mails: canosa@ fisica.unlp.edu.ar (N.C.); leocili@gmail.com (L.C.)

* Author to whom correspondence should be addressed; E-Mail: rossigno@ fisica.unlp.edu.ar;

Tel.:+54-221-4247-201; Fax:+54-221-4252-006.

Academic Editor: Demosthenes Ellinas, Giorgio Kaniadakis, Jiannis Pachos and Antonio M. Scarfone

Received: 14 January 2015 / Accepted: 19 March 2015 / Published: 26 March 2015

\begin{abstract}
We examine the behavior of quantum correlations of spin pairs in a finite anisotropic $X Y$ spin chain immersed in a transverse magnetic field, through the analysis of the quantum discord and the conventional and quadratic one-way information deficits. We first provide a brief review of these measures, showing that the last ones can be obtained as particular cases of a generalized information deficit based on general entropic forms. All of these measures coincide with an entanglement entropy in the case of pure states, but can be non-zero in separable mixed states, vanishing just for classically correlated states. It is then shown that their behavior in the exact ground state of the chain exhibits similar features, deviating significantly from that of the pair entanglement below the critical field. In contrast with entanglement, they reach full range in this region, becoming independent of the pair separation and coupling range in the immediate vicinity of the factorizing field. It is also shown, however, that significant differences between the quantum discord and the information deficits arise in the local minimizing measurement that defines them. Both analytical and numerical results are provided.
\end{abstract}

Keywords: quantum correlations; entropic measures; quantum discord; information deficit; spin systems 


\section{Introduction}

The investigation of non-classical correlations in mixed states of composite quantum systems has attracted strong attention in recent years. While in pure states, such correlations can be identified with entanglement [1-7], in the case of mixed states, separable (unentangled) states, defined in general as convex mixtures of product states [8], i.e., as states that can be generated by local operations and classical communication (LOCC), may still exhibit non-classical features. The latter emerge from the possible non-commutativity of the different products and lead, for instance, to a finite value of the quantum discord [9-13] and other recently introduced related quantifiers of quantum correlations [14,15]. These quantifiers include the one-way information deficit [12,13,16-18], the geometric discord [19], generalized entropic measures [20,21] and, more recently, the local quantum uncertainty [22,23] and the trace distance discord [24-27]. While entanglement is certainly necessary for quantum teleportation [28] and for an exponential speed-up in pure state-based quantum computation $[29,30]$, interest in these new measures has been triggered by the existence of mixed state-based quantum algorithms, like that of [31], able to achieve an exponential speedup over the best classical algorithms for a certain task, with vanishing entanglement [32], but finite quantum discord [33]. Additionally, various operational interpretations of the quantum discord and other related measures have been provided [15,22,26,34-39].

In this article, we will concentrate on the quantum discord [9-13] and the generalized entropic measures of [20], which include as particular cases the von Neumann-based one-way information deficit [12,13,16-18] and the geometric discord [19] and which represent a generalized information deficit. The quantum discord, as well as all other related measures require a rather complex minimization over a local measurement or operation, which has limited their applicability to small systems or special states. The optimization problem for the quantum discord was in fact recently shown to be NP complete [40]. The advantage of the generalized entropic formalism is, first, the possibility of using simpler entropic forms, like the linear entropy, which, as will be discussed in Section 2, enables an easier evaluation (it does not require the diagonalization of the density matrix) and a more direct experimental access (it can be determined without a full state tomography). This entails that an explicit solution of the associated optimization problem for certain states can be achieved. The generalized formalism also allows one to identify some universal properties, i.e., valid for any entropic form (and not just for a particular choice of entropy) satisfied by the post-measurement state.

We first provide in Section 2 an overview of the main concepts and properties associated with these measures. We then apply these measures to examine the quantum correlations of spin pairs in the exact ground state of finite spin $1 / 2$ chains with $X Y$-type couplings in a transverse magnetic field, through their entanglement, quantum discord and information deficit. All separations between the pairs are considered. Several important studies of the quantum discord in spins chains have been made [41-52], but the relation to the generalized information deficit and the differences between their optimizing measurements in these spin pairs have not yet been analyzed in detail. We have recently investigated these aspects for an $X X$ spin chain in [51] and will here extend this analysis to the anisotropic $X Y$ case. It is first shown that in contrast with the pair entanglement, the quantum discord and the information deficit exhibit, for the exact ground state of these chains, common features, such as 
an appreciable finite value below the critical field, for all separations. Moreover, they approach a finite common non-zero value [44] at the remarkable factorizing field [49,50,53-60] that these chains can exhibit in the anisotropic case. On the other hand, we will also show that important differences between the quantum discord on the one side and the standard and generalized information deficit on the other side do arise in the minimizing local spin measurement that defines them. While in the quantum discord, the direction of the latter is always orthogonal to the transverse field, in the other measures, it exhibits a perpendicular to parallel transition as the field increases, which is present for all separations and which reflects significant qualitative changes in the reduced state of the pair. This difference indicates a distinct response of the minimizing measurement of these quantities to the onset of quantum correlations.

\section{Measures of Quantum Correlations}

\subsection{Quantum Entanglement}

We start by providing a brief overview of the basic notions. A pure state $\left|\Psi_{A B}\right\rangle$ of a bipartite system $A+B$ is separable iff (if and only if) it is a product state $\left|\Psi_{A}\right\rangle\left|\Psi_{B}\right\rangle$. Otherwise, it is entangled. The Schmidt decomposition [5]:

$$
\left|\Psi_{A B}\right\rangle=\sum_{k=1}^{n_{s}} \sqrt{p_{k}}\left|k_{A}\right\rangle\left|k_{B}\right\rangle
$$

where $\left|k_{A(B)}\right\rangle$ denote orthonormal states for subsystem $A(B)$ and $p_{k} \geq 0, \sum_{k=1}^{n_{s}} p_{k}=1$, allows one to easily distinguish separable pure states $\left(n_{s}=1\right)$ from entangled states $\left(n_{s} \geq 2\right)$. Here, $n_{s}$ is the Schmidt rank of $\left|\Psi_{A B}\right\rangle\left(n_{s} \leq \operatorname{Min}\left[d_{A}, d_{B}\right]\right.$, with $d_{A(B)}$ the Hilbert space dimensions of $\left.A(B)\right)$. Pure sate entanglement can be measured by the entanglement entropy [3,4]:

$$
E(A, B)=S\left(\rho_{A}\right)=S\left(\rho_{B}\right)=-\sum_{k=1}^{n_{s}} p_{k} \log p_{k}
$$

where $\rho_{A(B)}=\operatorname{Tr}_{B(A)} \rho_{A B}=\sum_{k=1}^{n_{s}} p_{k}\left|k_{A(B)}\right\rangle\left\langle k_{A(B)}\right|$, with $\rho_{A B}=\left|\Psi_{A B}\right\rangle\left\langle\Psi_{A B}\right|$, are the reduced states of $A(B)$ and $S(\rho)=-\operatorname{Tr} \rho \log \rho$ is the von Neumann entropy. We will set in what follows $\log p \equiv$ $\log _{2} p$, such that $E(A, B)=1$ for a maximally entangled two-qubit state $\left(n_{s}=2, p_{1}=p_{2}=1 / 2\right.$ ).

On the other hand, a general mixed state $\rho_{A B}\left(\rho_{A B} \geq 0, \operatorname{Tr} \rho_{A B}=1\right)$ of a bipartite system $A+B$ is separable iff it can be expressed as a convex mixture of product states [8]:

$$
\rho_{A B} \text { separable } \Leftrightarrow \rho_{A B}=\sum_{\alpha} p_{\alpha} \rho_{A}^{\alpha} \otimes \rho_{B}^{\alpha} \quad p_{\alpha}>0
$$

where $\sum_{\alpha} p_{\alpha}=1$ and $\rho_{A(B)}^{\alpha}$ denote mixed states for subsystem $A(B)$. Otherwise, it is entangled. The meaning is that a separable state can be created by LOCC, i.e., Alice prepares a state $\rho_{A}^{\alpha}$ with probability $p_{\alpha}$ and tells Bob to prepare a partner state $\rho_{B}^{\alpha}$.

For pure states $\rho_{A B}=\left|\Psi_{A B}\right\rangle\left\langle\Psi_{A B}\right|$, Equation (3) is equivalent to the previous definition $\left(\left|\Psi_{A B}\right\rangle=\right.$ $\left|\Psi_{A}\right\rangle\left|\Psi_{B}\right\rangle$ ), but in the case of mixed states, product states $\rho_{A B}=\rho_{A} \otimes \rho_{B}$ are just a very particular case of separable states. The latter also include: (a) classically-correlated states, i.e., states diagonal in a standard product basis $\left\{|i j\rangle \equiv\left|i_{A}\right\rangle\left|j_{B}\right\rangle\right\}$,

$$
\rho_{A B}=\sum_{i, j} p_{i j}\left|i_{A}\right\rangle\left\langle i_{A}|\otimes| j_{B}\right\rangle\left\langle j_{B}\right|, \quad p_{i j} \geq 0
$$


where $\sum_{i, j} p_{i j}=1$ and $\left|i_{A(B)}\right\rangle$ are orthonormal states of $A(B)$; (b) classically-correlated states from one of the subsystems, say $B$, which are of the form:

$$
\rho_{A B}=\sum_{j} p_{j} \rho_{A / j} \otimes\left|j_{B}\right\rangle\left\langle j_{B}\right|, \quad p_{j} \geq 0
$$

where $\sum_{j} p_{j}=1$ and $\rho_{A / j}$ are states of $A$, which are then diagonal in a conditional product basis $\left\{\left|i_{j} j\right\rangle \equiv\left|i_{A / j}\right\rangle\left|j_{B}\right\rangle\right\}$ with $\left|i_{A / j}\right\rangle$ the eigenstates of $\rho_{A / j}$ (the case (4) recovered when all $\rho_{A / j}$ commute); and (c) convex mixtures of product states, which are not of the previous forms of (a) or (b). The latter typically possess entangled eigenstates. For this reason, it is much more difficult to determine whether a mixed state is separable or entangled. The well-known positive partial transpose criterion $[61,62]$ $\left(\rho_{A B}^{t_{B}} \geq 0\right.$, with $\rho_{i j, k l}^{t_{B}}=\rho_{i l, k j}$ for $\left.\rho_{i l k j}=\left\langle i l\left|\rho_{A B}\right| k j\right\rangle\right)$ provides a necessary criterion for separability, which is sufficient for two-qubit or qubit-qutrit states.

For mixed states, the marginal entropies $S\left(\rho_{A}\right), S\left(\rho_{B}\right)$ no longer provide a measure of entanglement. Instead, it is possible to use the entanglement of formation [63], defined through the convex roof extension of the pure state definition:

$$
E(A, B)=\underset{\sum_{\alpha} p_{\alpha}\left|\Psi_{A B}^{\alpha}\right\rangle\left\langle\Psi_{A B}^{\alpha}\right|=\rho_{A B}}{\operatorname{Min}} S\left(\rho_{A}^{\alpha}\right)
$$

where the minimization is over all decompositions of $\rho_{A B}$ as convex mixtures of pure states $\left(p_{\alpha} \geq 0, \sum_{\alpha} p_{\alpha}=1\right)$ and $S\left(\rho_{A}^{\alpha}\right)=S\left(\rho_{B}^{\alpha}\right)$ is the entanglement entropy of the pure state $\left|\Psi_{A B}^{\alpha}\right\rangle$. Equation (6) vanishes iff $\rho_{A B}$ is separable and reduces to the entanglement entropy (2) for pure states. It is an entanglement monotone [64], i.e., it does not increase by LOCC, staying unaltered under local unitary operations $\rho_{A B} \rightarrow U_{A} \otimes U_{B} \rho_{A B} U_{A}^{\dagger} \otimes U_{B}^{\dagger}$. Its evaluation is, however, difficult in general. A general analytic expression has been derived just for the two-qubit case $[65,66]$, which will be specified in Section 3.

While the marginal entropies are no longer entanglement indicators, it can still be shown [67] that if $S\left(\rho_{A}\right)>S\left(\rho_{A B}\right)$ or $S\left(\rho_{B}\right)>S\left(\rho_{A B}\right), \rho_{A B}$ is entangled, i.e.,

$$
\rho_{A B} \text { separable } \Rightarrow S\left(\rho_{A}\right) \leq S\left(\rho_{A B}\right), \quad S\left(\rho_{B}\right) \leq S\left(\rho_{A B}\right)
$$

Equation (7) provides an entropic criterion for separability [67] (necessary, but not sufficient in general), which can be also extended to more general entropic forms [68-70] and which will be invoked in Section 2.3.

\subsection{Quantum Discord}

For the classically-correlated states (4) or in general (5), there is a complete local measurement on $B$ that leaves the state unaltered. This is not the case for entangled states, nor for separable states not of the form of (4) or (5). Let us recall here that a general positive operator valued measurement (POVM) [5] on system $A+B$ is defined by a set of operators $\left\{M_{j}\right\}$ satisfying $\sum_{j} M_{j}^{\dagger} M_{j}=I_{A B} \equiv I_{A} \otimes I_{B}$, such that the probability of outcome $j$ and the joint state after such an outcome are:

$$
p_{j}=\operatorname{Tr} \rho_{A B} M_{j}, \quad \rho_{A B / j}^{\prime}=M_{j} \rho_{A B} M_{j}^{\dagger} / p_{j}
$$


The post-measurement state if the outcome is unknown is then:

$$
\rho_{A B}^{\prime}=\sum_{j} p_{j} \rho_{A B / j}^{\prime}=\sum_{j} M_{j} \rho_{A B} M_{j}^{\dagger}
$$

Standard projective measurements correspond to the case where the $M_{j}$ are orthogonal projectors $\left(M_{k} M_{j}=\delta_{j k} M_{j}\right)$, while a local measurement on $B$ corresponds to $M_{j}=I_{A} \otimes M_{j}^{B}$. By a complete local measurement on $B$, we will mean one based on rank one orthogonal projectors $M_{j}^{B}=P_{j}^{B}$. It is then apparent that the states (4) and (5) remain unchanged $\left(\rho_{A B}^{\prime}=\rho_{A B}\right)$ after a local measurement on $B$ based on the projectors $P_{j}^{B}=\left|j_{B}\right\rangle\left\langle j_{B}\right|$. For the states (4) (but not necessarily (5)), there is also a local measurement on $A$ (that based on the projectors $\left|i_{A}\right\rangle\left\langle i_{A}\right|$ ), which leaves them unchanged.

The quantum discord [9-13] is a measure of quantum correlations, which, unlike the entanglement of formation, can distinguish the classically-correlated states (5) from the rest of separable states: It vanishes iff $\rho_{A B}$ is of the form of (4) or (5), being positive in the other separable states (c), and reduces to the entanglement entropy (2) in the case of pure states. It can be defined as the minimum difference between two distinct quantum versions of the mutual information, or equivalently, of the conditional entropy:

$$
D(A \mid B)=\operatorname{Min}_{M_{B}}\left[I(A, B)-I\left(A, B_{M_{B}}\right)\right]=\operatorname{Min}_{M_{B}} S\left(A \mid B_{M_{B}}\right)-S(A \mid B)
$$

where the minimization is over all local measurements $M_{B}$ on $B$ and:

$$
I(A, B)=S\left(\rho_{A}\right)-S(A \mid B), S(A \mid B)=S\left(\rho_{A B}\right)-S\left(\rho_{B}\right)
$$

are, respectively, the standard quantum mutual information and conditional entropy, while:

$$
I\left(A, B_{M_{B}}\right)=S\left(\rho_{A}\right)-S\left(A \mid B_{M_{B}}\right), \quad S\left(A \mid B_{M_{B}}\right)=\sum_{j} p_{j} S\left(\rho_{A / j}\right)
$$

are the mutual information and conditional entropy after the local measurement $M_{B}$, with $\rho_{A / j}=$ $\operatorname{Tr}_{B} \rho_{A B / j}^{\prime}$ the reduced state of $A$ after outcome $j$. Equation (10) is always non-negative [9-11], a property that arises from the concavity of the conditional von Neumann entropy [71].

In the case of complete local projective measurements $M_{B}$, we have:

$$
S\left(A \mid B_{M_{B}}\right)=S\left(\rho_{A B}^{\prime}\right)-S\left(\rho_{B}^{\prime}\right)
$$

where $\rho_{B}^{\prime}=\operatorname{Tr}_{A} \rho_{A B}^{\prime}$ and $\rho_{A B}^{\prime}$ is the post-measurement state (9). It is then apparent that if the state is of the form (4) or (5), a measurement $M_{B}$ based on the projectors $P_{j}^{B}=\left|j_{B}\right\rangle\left\langle j_{B}\right|$ leads to $S\left(A \mid B_{M_{B}}\right)=$ $S(A \mid B)$ and, hence, $D(A \mid B)=0$. For all other states (i.e., entangled states or separable states not of the form of (4) or (5)), $D(A \mid B)>0$. In the case of pure states, $S\left(\rho_{A B}\right)=0$, while $S\left(A \mid B_{M_{B}}\right)=0$ if $M_{B}$ is any complete local measurement, entailing $D(A \mid B)=S\left(\rho_{B}\right)=E(A, B)$. For mixed states, the quantum discord can be related to the entanglement of formation $E(A, C)$ with a third system $C$ purifying the whole system [35-38].

The mutual information $I(A, B)$ is a measure of all correlations between $A$ and $B$, being non-negative and vanishing just for product states $\rho_{A B}=\rho_{A} \otimes \rho_{B}$. The bracket in (10) can then be interpreted as the difference between all correlations (classical + quantum) present in the original state minus the 
classical correlations left after the local measurement on $B$, which leaves then the quantum correlations. The evaluation of Equation (10) is, nevertheless, difficult in the general case, being in fact an NP complete problem [40] due to the minimization over all possible local measurements $M_{B}$. Nonetheless, the minimum is always attained for measurements based on rank one projectors $P_{j}^{B}$, not necessarily orthogonal $[15,72,73]$.

\subsection{Information Deficit}

The one-way information deficit can be considered as an alternative measure of quantum correlations, with basic properties similar to those of the quantum discord. It can be defined as $[12,13,16-18,20]$ :

$$
I(A \mid B)=\operatorname{Min}_{M_{B}} S\left(\rho_{A B}^{\prime}\right)-S\left(\rho_{A B}\right)
$$

where $\rho_{A B}^{\prime}$ is the post-measurement state (9) and $M_{B}$ is here restricted to complete local projective measurements on $B$, such that $\rho_{A B}^{\prime}$ is of the form of (5). Like the quantum discord, Equation (14) is a non-negative quantity, which also vanishes just for the states (4) or (5) and which also reduces to the entanglement entropy (2) in the case of pure states. These properties will be shown below in a more general context, although they are also apparent from the alternative expression:

$$
I(A \mid B)=\operatorname{Min}_{M_{B}} S\left(\rho_{A B} \| \rho_{A B}^{\prime}\right)
$$

where $S(\rho \| \sigma)=\operatorname{Tr} \rho(\log \rho-\log \sigma)$ is the relative entropy $[71,74]$ and a quantity satisfying $S(\rho \| \sigma) \geq 0$, with $S(\rho \| \sigma)=0$ iff $\rho=\sigma$. Equation (15) can be shown by noting that $\rho_{A B}^{\prime}$ is the diagonal part of $\rho_{A B}$ in the basis defined by the projective measurement (the minimization in (15) can in fact be extended to all $\rho_{A B}^{\prime}$ of the form of (5) [20]). Nevertheless, differences with the quantum discord may arise in the minimizing measurement, as discussed in the next section. We also note that if the minimizing measurement of $D(A \mid B)$ is projective and in the basis of eigenstates of $\rho_{B}$, then $\rho_{B}^{\prime}=\rho_{B}$ and Equations (10)-(13) lead to $D(A \mid B)=I(A \mid B)$. Otherwise, $D(A \mid B) \leq I(A \mid B)$, since for projective measurements, Equations (10)-(13) imply $D(A \mid B) \leq S\left(\rho_{A B}^{\prime}\right)-S\left(\rho_{A B}\right)-\left[S\left(\rho_{B}^{\prime}\right)-S\left(\rho_{B}\right)\right] \leq$ $S\left(\rho_{A B}^{\prime}\right)-S\left(\rho_{A B}\right)$.

Equation (14) admits a simple interpretation in terms of the entanglement generated between the system and a measuring apparatus $M$ performing the complete local measurement [18]. The measurement on the local basis $\left\{\left|i_{B}\right\rangle\right\}$ can be represented through a unitary operator $U_{B M}$ satisfying $U_{B M}\left|j_{B} 0_{M}\right\rangle=\left|j_{B} j_{M}\right\rangle$, where $\left|0_{M}\right\rangle$ is the initial state of the apparatus and $\left\{\left|j_{M}\right\rangle\right\}$ an orthogonal basis of $M$, such that:

$$
\rho_{A B}^{\prime}=\operatorname{Tr}_{M} \rho_{A B M}^{\prime}, \quad \rho_{A B M}^{\prime}=\left(I_{A} \otimes U_{B M}\right)\left(\rho_{A B} \otimes\left|0_{M}\right\rangle\left\langle 0_{M}\right|\right)\left(I_{A} \otimes U_{B M}^{\dagger}\right)
$$

Since $S\left(\rho_{A B}\right)=S\left(\rho_{A B} \otimes\left|0_{M}\right\rangle\left\langle 0_{M}\right|\right)=S\left(\rho_{A B M}^{\prime}\right)$, it is seen that Equation (14) is the difference between the entropy of the subsystem $A B$ and that of the total system $A B M$ after the measurement, and according to Equation (7), such a difference can be positive only if there is entanglement between $A B$ and $M$. Thus, a positive $I(A \mid B)$ indicates that entanglement between $A B$ and $M$ is generated by any complete local measurement $M_{B}$. On the other hand, if $I(A \mid B)=0$, then $\rho_{A B}$ is of the form of (5) and for a measurement in the basis $\left\{\left|j_{B}\right\rangle\right\}, \rho_{A B M}^{\prime}=\sum_{j} p_{j} \rho_{A / j} \otimes\left|j_{B} j_{M}\right\rangle\left\langle j_{B} j_{M}\right|$ is clearly separable, so that no 
entanglement is generated by this measurement. It can be shown [18] that Equation (14) coincides in fact with the minimum distillable entanglement between $A B$ and $M$ generated by the complete local measurement on $B$. A similar interpretation for the quantum discord in terms of the minimum partial distillable entanglement can also be obtained [18]. Other operational interpretations can be found in [26,34,36-39].

\subsection{Generalized Information Deficit}

It is possible in principle to extend Equation (14) to more general entropic forms, since in contrast with the quantum discord (10), its positivity is not related to specific properties of the von Neumann entropy $S(\rho)$, as shown below. We consider here generalized entropies of the form [75]:

$$
S_{f}(\rho)=\operatorname{Tr} f(\rho)
$$

where $\operatorname{Tr} f(\rho)=\sum_{i} f\left(p_{i}\right)$, with $p_{i}$ the eigenvalues of $\rho$ and $f(p)$ a smooth strictly concave real function defined for $p \in[0,1]$ and satisfying $f(0)=f(1)=0$. These entropies fulfill the same basic properties as the von Neumann entropy, with the exception of additivity: we have $S_{f}(\rho) \geq 0$, with $S_{f}(\rho)=0$ iff $\rho$ is a pure state $\left(\rho^{2}=\rho\right)$, while all $S_{f}(\rho)$ are maximum for the maximally mixed state $\rho=I / d$, where $d=\operatorname{Tr} I$ is the Hilbert space dimension of the system. Moreover, they are strictly concave, i.e., $S_{f}\left(\sum_{\alpha} p_{\alpha} \rho_{\alpha}\right) \geq \sum_{\alpha} p_{\alpha} S_{f}\left(\rho_{\alpha}\right)$, for $p_{\alpha}>0, \sum_{\alpha} p_{\alpha}=1$, with equality iff all $\rho_{\alpha}$ are coincident. The von Neumann entropy is obviously recovered for $f(\rho)=-\rho \log \rho$.

Concavity of $S_{f}(\rho)$ implies the fundamental majorization property:

$$
\rho^{\prime} \prec \rho \Rightarrow S_{f}\left(\rho^{\prime}\right) \geq S_{f}(\rho)
$$

where $\rho^{\prime} \prec \rho$ indicates that $\rho^{\prime}$ is majorized by $\rho[71,76,77]$ (also denoted as $\rho^{\prime}$ more mixed than $\rho$ ):

$$
\rho^{\prime} \prec \rho \Leftrightarrow \sum_{j=1}^{i} p_{j}^{\prime} \leq \sum_{j=1}^{i} p_{j}, \quad i=1, \ldots, d-1
$$

where $p_{j}, p_{j}^{\prime}$ denote the eigenvalues of $\rho$ and $\rho^{\prime}$ sorted in decreasing order (equality in (19) obviously holds for $i=d$ ). If the dimensions of $\rho$ and $\rho^{\prime}$ differ, Equation (18) still holds (for $\left.f(0)=0\right)$ after completing with zeros the smallest set of eigenvalues. Conversely, while the reverse of Equation (18) does not necessarily hold, indicating that majorization provides a more strict concept of mixedness or disorder than that defined by a single choice of entropy, it does hold if $S_{f}\left(\rho^{\prime}\right) \geq S_{f}(\rho) \forall f$ of the previous form $[69,70]$ :

$$
S_{f}\left(\rho^{\prime}\right) \geq S_{f}(\rho) \forall S_{f} \Rightarrow \rho^{\prime} \prec \rho
$$

Equation (18) remains actually valid for more general entropic forms (like increasing functions $F\left(S_{f}\right)$ of $S_{f}$ or, in general, Schur concave functions [76,77]), but Equation (20) indicates that the forms (17) are already sufficient to capture majorization. Among the various properties implied by majorization, we mention that for states with the same dimension, $\rho^{\prime} \prec \rho$ iff $\rho^{\prime}$ is a convex mixture of unitary transformations of $\rho[71,76,77]$, i.e., iff $\rho^{\prime}=\sum_{\alpha} p_{\alpha} U_{\alpha} \rho U_{\alpha}^{\dagger}$, with $U_{\alpha}$ unitary and $p_{\alpha} \geq 0$.

Now, for any projective measurement (local or non-local) performed on the system $A+B$, it can be easily shown that $S_{f}\left(\rho_{A B}^{\prime}\right) \geq S_{f}\left(\rho_{A B}\right) \forall S_{f}$, i.e.,

$$
\rho_{A B}^{\prime} \prec \rho_{A B}
$$


The reason is that the post measurement state $\rho_{A B}^{\prime}$ conserves just the diagonal elements $p_{\nu}^{\prime}=\left\langle\nu^{\prime}\left|\rho_{A B}\right| \nu^{\prime}\right\rangle$ of $\rho_{A B}$ in a certain orthonormal basis $\left\{\left|\nu^{\prime}\right\rangle\right\}$ determined by the projectors, and hence, $S_{f}\left(\rho_{A B}^{\prime}\right)=$ $\sum_{\nu} f\left(p_{\nu}^{\prime}\right)=\sum_{\nu} f\left(\sum_{\mu}\left|\left\langle\mu \mid \nu^{\prime}\right\rangle\right|^{2} p_{\mu}\right) \geq \sum_{\mu, \nu}\left|\left\langle\mu \mid \nu^{\prime}\right\rangle\right|^{2} f\left(p_{\mu}\right)=S_{f}\left(\rho_{A B}\right)$, where $p_{\mu}$ and $|\mu\rangle$ denote here the eigenvalues and eigenvectors of $\rho_{A B}$. This relation is not restricted to rank one projectors (just choose an orthonormal basis $\left\{\left|\nu^{\prime}\right\rangle\right\}$ where $\rho_{A B}^{\prime}$ is diagonal), so that it holds for local projective measurements. Equation (21) remains actually valid for any measurement satisfying $\sum_{j} M_{j} M_{j}^{\dagger}=I_{A B}$, i.e., which leaves the maximally-mixed state $I_{A B} / d_{A B}$ unchanged [20].

Note also that the strict concavity of $S_{f}$ implies $S_{f}\left(\rho_{A B}^{\prime}\right)=S_{f}\left(\rho_{A B}\right)$ iff $\rho_{A B}^{\prime}=\rho_{A B}$, as is apparent from the previous demonstration. In fact, if the off diagonal elements of $\rho_{A B}$ in the measured basis are sufficiently small, a second order expansion of $S_{f}\left(\rho_{A B}\right)$ leads to [20]:

$$
S_{f}\left(\rho_{A B}^{\prime}\right)-S_{f}\left(\rho_{A B}\right) \approx \sum_{\mu<\nu} \frac{f^{\prime}\left(p_{\mu}^{\prime}\right)-f^{\prime}\left(p_{\nu}^{\prime}\right)}{p_{\nu}^{\prime}-p_{\mu}^{\prime}}\left|\left\langle\nu^{\prime}\left|\rho_{A B}\right| \mu^{\prime}\right\rangle\right|^{2}
$$

where the fraction is always positive due to the strict concavity of $f$ (and should be replaced by its limit $-f^{\prime \prime}\left(p_{\mu}^{\prime}\right)$ if $p_{\nu}^{\prime} \rightarrow p_{\mu}^{\prime}$ ). Equation (22) is essentially the square of a weighted norm of the off-diagonal elements of $\rho_{A B}$ in the measured basis (i.e., of those lost in the measurement) and is therefore non-negative, vanishing (if $f^{\prime \prime}(p)<0 \forall p \in(0,1)$ ) only if all off-diagonal elements are zero.

We may then define the quantity $[20,21]$ :

$$
I_{f}(A \mid B)=\operatorname{Min}_{M_{B}} S_{f}\left(\rho_{A B}^{\prime}\right)-S_{f}\left(\rho_{A B}\right)
$$

where the minimization is again over all complete local measurements on $B$. Equation (23) is non-negative, due to Equation (21), and vanishes iff $\rho_{A B}^{\prime}=\rho_{A B}$, i.e., iff $\rho_{A B}$ is already of the classically-correlated form of (4) or (5). It therefore vanishes only for the states with zero quantum discord. It obviously also remains invariant under local unitary operations.

In the case of pure states, it can be shown [20] that the minimum of Equation (23) is always attained for a measurement in the basis $\left\{\left|k_{B}\right\rangle\right\}$ determined by the Schmidt decomposition (1), i.e., in the basis formed by the eigenstates of $\rho_{B}$, which leads to:

$$
I_{f}(A \mid B)=S_{f}\left(\rho_{A}\right)=S_{f}\left(\rho_{B}\right)=\sum_{k=1}^{n_{s}} f\left(p_{k}\right), \quad\left(\rho_{A B} \text { pure }\right)
$$

It therefore reduces to the generalized entanglement entropy $S_{f}\left(\rho_{A}\right)=S_{f}\left(\rho_{B}\right)$ of the pure state. The entanglement entropy can then be identified with the minimum information loss due to a local measurement [20]. It is apparent that for pure states, $I_{f}(A \mid B)=I_{f}(B \mid A)$, a property which does not hold in the general case.

In the case of the von Neumann entropy, $I_{f}(A \mid B)$ becomes the standard information deficit (14), and Equation (24) implies that for pure states, it will coincide with the standard (von Neumann) entanglement entropy, like the quantum discord. Nevertheless, an important difference arises in the minimizing measurement, since that for the latter becomes undetermined in the case of pure states (it can be any measurement based on rank one projectors [72,73]), whereas all $I_{f}(A \mid B)$, including $I(A \mid B)$, require a measurement in the basis $\left\{\left|k_{B}\right\rangle\right\}$, which is fully undetermined only in the case of maximally-mixed marginals. 
Like the standard information deficit, $I_{f}(A \mid B)$ is also an indicator of the minimum entanglement between the system and the measurement apparatus $M$ generated by a complete local measurement. The von Neumann entropic criterion for separability (7) can actually be extended to any $S_{f}[69,70]$ :

$$
\rho_{A B} \text { separable } \Rightarrow S_{f}\left(\rho_{A}\right) \leq S_{f}\left(\rho_{A B}\right), \quad S_{f}\left(\rho_{B}\right) \leq S_{f}\left(\rho_{A B}\right)
$$

The validity of Equation (25) for all $S_{f}$ is stronger than the von Neumann-based criterion (23) [69,70] and equivalent to the disorder criterion of separability [68] ( $\rho_{A B}$ separable $\left.\Rightarrow \rho_{A B} \prec \rho_{A(B)}\right)$. By the same arguments given below, Equation (16), it follows that a positive $I_{f}(A \mid B)$, i.e., $S_{f}\left(\rho_{A B}^{\prime}\right)>S_{f}\left(\rho_{A B}\right)=$ $S_{f}\left(\rho_{A B M}^{\prime}\right)$, is indicating the existence of entanglement between $A B$ and $M$ after any complete local projective measurement on $B$.

\subsection{Minimizing Measurement}

Equation (24) reflects a universal property exhibited by the local measurement minimizing $I_{f}(A \mid B)$ for pure states: it is the same for all $S_{f}$. Such a measurement, i.e., a measurement in the basis $\left\{\left|k_{B}\right\rangle\right\}$ determined by the Schmidt decomposition of the pure state, is also optimum, for all $S_{f}$, for the mixture of the pure state with the maximally-mixed state [20],

$$
\rho_{A B}=q\left|\Psi_{A B}\right\rangle\left\langle\Psi_{A B}\right|+(1-q) I_{A B} / d_{A B}, \quad q \in[0,1]
$$

These states exhibit then an unambiguous least disturbing local measurement, in the sense that it minimizes all $I_{f}(A \mid B)$ and leads to a "least mixed" post-measurement state:

$$
\rho_{A B}^{\prime}=q \sum_{k=1}^{n_{s}} p_{k}\left|k_{A}\right\rangle\left\langle k_{A}|\otimes| k_{B}\right\rangle\left\langle k_{B}\right|+(1-q) I_{A B} / d_{A B}
$$

which majorizes any other post-measurement state emerging after a local measurement. This property does not hold for an arbitrary initial state $\rho_{A B}$.

In the general case, the projective measurement $M_{B}=\left\{\left|j_{B}\right\rangle\left\langle j_{B}\right|\right\}$ minimizing $I_{f}(A \mid B)$ may depend on the choice of entropy $S_{f}$. It can be shown that it must satisfy the necessary stationary condition [21]:

$$
\operatorname{Tr}_{A}\left[f^{\prime}\left(\rho_{A B}^{\prime}\right), \rho_{A B}\right]=0
$$

where $f^{\prime}$ denotes the derivative of $f$ and $\rho_{A B}^{\prime}$ is the post-measurement state (9). Equation (27) implies, explicitly, $\sum_{i}\left[f^{\prime}\left(p_{i j}^{\prime}\right)\left\langle i_{j} j\left|\rho_{A B}\right| i_{j} k\right\rangle-f^{\prime}\left(p_{i k}^{\prime}\right)\left\langle i_{k} j\left|\rho_{A B}\right| i_{k} k\right\rangle\right]=0$, where $p_{i j}^{\prime}=\left\langle i_{j} j\left|\rho_{A B}\right| i_{j} j\right\rangle$ and $\left|i_{j} j\right\rangle=$ $\left|i_{A / j}\right\rangle\left|j_{B}\right\rangle$, with $\left|i_{j / A}\right\rangle$ the eigenstates of $\rho_{A / j}$. The minimizing measurement basis will not coincide in general with the eigenstates of $\rho_{B}$, even though this holds for certain states, like pure states and the mixtures (26). Equation (27) shows that the eigenstates of $\rho_{B}$ will be stationary for any state $\rho_{A B}$ where the non-zero off-diagonal elements are of the form $\left\langle i j\left|\rho_{A B}\right| k l\right\rangle$ with $i \neq k$ and $j \neq l$, where $|i j\rangle \equiv\left|i_{A}\right\rangle\left|j_{B}\right\rangle$ and $\left|i_{A}\right\rangle,\left|j_{B}\right\rangle$ are the eigenstates of $\rho_{A}$ and $\rho_{B}$ respectively [21].

In the case of the quantum discord and for $M_{B}$ restricted to complete local projective measurements, Equation (27) is to be replaced by (here $f^{\prime}(\rho)=-\log \rho$ ) [21]:

$$
\operatorname{Tr}_{A}\left[f^{\prime}\left(\rho_{A B}^{\prime}\right), \rho_{A B}\right]-\left[f^{\prime}\left(\rho_{A B}^{\prime}\right), \rho_{B}\right]=0
$$


More explicit expressions can be obtained for a two-qubit system, where we may write a general state as:

$$
\rho_{A B}=\frac{1}{4}\left(I_{A B}+\boldsymbol{r}_{A} \cdot \boldsymbol{\sigma}_{A}+\boldsymbol{r}_{B} \cdot \boldsymbol{\sigma}_{B}+\boldsymbol{\sigma}_{A}^{t} J \boldsymbol{\sigma}_{B}\right)
$$

where $\sigma_{A}=\boldsymbol{\sigma} \otimes I, \sigma_{B}=I \otimes \boldsymbol{\sigma}$, with $\boldsymbol{\sigma}^{t}=\left(\sigma_{x}, \sigma_{y}, \sigma_{z}\right)$ the Pauli operators, and $I_{A B}=I \otimes I$ the identity. Since $\operatorname{Tr} \sigma_{\mu}=0$ and $\operatorname{Tr} \sigma_{\mu} \sigma_{\nu}=2 \delta_{\mu \nu}$ for $\mu, \nu=x, y, z$, we have $\left(\langle O\rangle \equiv \operatorname{Tr} \rho_{A B} O\right)$ :

$$
\boldsymbol{r}_{A(B)}=\left\langle\boldsymbol{\sigma}_{A(B)}\right\rangle, \quad J=\left\langle\boldsymbol{\sigma}_{A} \boldsymbol{\sigma}_{B}^{t}\right\rangle
$$

A complete projective measurement on $B$ corresponds to a spin measurement along the direction of a unit vector $\boldsymbol{k}$, represented by projectors $P_{ \pm \boldsymbol{k}}=\frac{1}{2}(I \pm \boldsymbol{k} \cdot \boldsymbol{\sigma})$. After this measurement, Equation (29) becomes:

$$
\rho_{A B}^{\prime}=\frac{1}{4}\left[I+\boldsymbol{r}_{A} \cdot \boldsymbol{\sigma}_{A}+\left(\boldsymbol{r}_{B} \cdot \boldsymbol{k}\right) \boldsymbol{k} \cdot \boldsymbol{\sigma}_{B}+\left(\boldsymbol{\sigma}_{A}^{t} J \boldsymbol{k}\right) \boldsymbol{k} \cdot \boldsymbol{\sigma}_{B}\right]
$$

Equation (27) leads then to the explicit equation [21]:

$$
\alpha_{1} \boldsymbol{r}_{B}+\alpha_{2} J^{t} \boldsymbol{r}_{A}+\alpha_{3} J^{t} J \boldsymbol{k}=\lambda \boldsymbol{k}
$$

where $\left(\alpha_{1}, \alpha_{2}, \alpha_{3}\right)=\frac{1}{4} \sum_{\mu, \nu= \pm 1} f^{\prime}\left(p_{\mu \nu}^{\prime}\right)\left(\nu, \frac{\mu \nu}{\left|\boldsymbol{r}_{A}+J \boldsymbol{k}\right|}, \frac{\mu}{\left|\boldsymbol{r}_{A}+J \boldsymbol{k}\right|}\right), p_{\mu \nu}^{\prime}=\frac{1}{4}\left(1+\nu \boldsymbol{r}_{B} \cdot \boldsymbol{k}+\mu\left|\boldsymbol{r}_{A}+\nu J \boldsymbol{k}\right|\right)$ are the eigenvalues of $\rho_{A B}^{\prime}$, with $\mu, \nu= \pm 1$, and $\lambda$ is a proportionality factor. In the case of the quantum discord, Equation (28) leads to a similar equation, with $f(p) \rightarrow-p \log p$ and $\alpha_{1} \rightarrow \alpha_{1}-\frac{1}{2} \log p_{-}^{\prime} / p_{+}^{\prime}$, where $p_{ \pm}^{\prime}=\frac{1}{2}\left(1+\boldsymbol{r}_{B} \cdot \boldsymbol{k}\right)$ are the eigenvalues of $\rho_{B}^{\prime}[21]$.

\subsection{Particular Cases}

One of the advantages of the generalized information deficit (23) is the possibility of using simple entropic forms, which can be more easily evaluated (and measured) than the von Neumann entropy. For instance, if $f(\rho)=2\left(\rho-\rho^{2}\right)$, Equation (17) becomes the so-called linear entropy:

$$
S_{2}(\rho)=2\left(1-\operatorname{Tr} \rho^{2}\right)
$$

which follows from the linear approximation $\ln \rho \approx \rho-I$ in the von Neumann entropy, but is actually a quadratic function of $\rho$, i.e., a linear function of the purity $P(\rho)=\operatorname{Tr} \rho^{2}$. It is the simplest entropic form, and its evaluation does not require the knowledge of the eigenvalues of $\rho$ (see Equation (39) below). Moreover, purity and, hence, $S_{2}(\rho)$, can be experimentally determined without a full-state tomography [78-80]. Equation (33) is actually the $q=2$ case of the Tsallis entropies [81,82], obtained for $f(\rho)=\frac{\rho-\rho^{q}}{1-2^{1-q}}$ :

$$
S_{q}(\rho)=\frac{1-\operatorname{Tr} \rho^{q}}{1-2^{1-q}}, \quad q>0
$$

Equation (34) approaches the von Neumann entropy $S(\rho)$ for $q \rightarrow 1$, being strictly concave for $q>0$. We have normalized (33) and (34), such that $S_{q}(\rho)=1$ for a maximally-mixed two-qubit state.

In the case (33), it is first seen that for post-measurements states $\rho_{A B}^{\prime}$ :

$$
S_{2}\left(\rho_{A B}^{\prime}\right)-S_{2}\left(\rho_{A B}\right)=2 \operatorname{Tr}\left(\rho_{A B}^{2}-\rho_{A B}^{\prime 2}\right)=2\left\|\rho_{A B}^{\prime}-\rho_{A B}\right\|^{2}
$$


where $\|O\|^{2}=\operatorname{Tr} O^{\dagger} O$. Hence, the local projective measurement minimizing $S_{2}(A \mid B)$, which is that maximizing the post-measurement purity $P\left(\rho_{A B}^{\prime}\right)$, leads to the post-measurement state with the minimum Hilbert-Schmidt distance to the original state. The associated deficit:

$$
I_{2}(A \mid B)=\operatorname{Min}_{M_{B}} S_{2}\left(\rho_{A B}^{\prime}\right)-S_{2}\left(\rho_{A B}\right)
$$

coincides, apart from a constant factor, with the geometric discord $[15,19,20]$. For pure states, $I_{2}(A \mid B)$ will then coincide with the linear marginal entropies:

$$
I_{2}(A \mid B)=S_{2}\left(\rho_{A}\right)=S_{2}\left(\rho_{B}\right)=2\left(1-\sum_{k=1}^{n_{s}} p_{k}^{2}\right)
$$

In two qubit systems, Equation (37) is just the squared concurrence $[65,66]$ of the pure state $\rho_{A B}$.

While as a measure, the geometric discord fails to satisfy some additional properties fulfilled by the quantum discord or the information deficit [83], it offers the enormous advantage of a simple analytic evaluation in qudit-qubit systems [19,21,84], as discussed below, also admitting through the purity a more direct experimental access. Moreover, Equation (22) shows that if $\rho_{A B}$ is close to the maximally-mixed state $I_{A B} / d_{A B}$, all $I_{f}(A \mid B)$ will become proportional to $I_{2}(A \mid B)$ [20], as in this case $\frac{f^{\prime}\left(p_{\mu}^{\prime}\right)-f^{\prime}\left(p_{\nu}^{\prime}\right)}{p_{\nu}^{\prime}-p_{\mu}^{\prime}} \approx-f^{\prime \prime}\left(\frac{1}{d_{A B}}\right)$ is nearly constant. In fact, all $S_{f}(\rho)$ are linearly related to $S_{2}(\rho)$ in this limit $[72,73]$.

Any state of a general system $A+B$ can be written in the form of (29), replacing the Pauli operators by a complete set of orthogonal operators $\sigma$ in $A$ and $B$ satisfying $\operatorname{Tr} \sigma_{\mu}=0$, $\operatorname{Tr} \sigma_{\mu} \sigma_{\nu}=d_{A(B)} \delta_{\mu \nu}$ :

$$
\rho_{A B}=\frac{1}{d_{A} d_{B}}\left(I_{A B}+\boldsymbol{r}_{A} \cdot \boldsymbol{\sigma}_{A}+\boldsymbol{r}_{B} \cdot \boldsymbol{\sigma}_{B}+\boldsymbol{\sigma}_{A}^{t} J \boldsymbol{\sigma}_{B}\right)
$$

where $\boldsymbol{r}_{A(B)}$ and $J$ (now a $d_{A} \times d_{B}$ matrix) are again given by Equation (30). The $S_{2}$ entropy can then be readily evaluated as:

$$
S_{2}\left(\rho_{A B}\right)=2\left[1-\frac{1}{d_{A} d_{B}}\left(1+\left|\boldsymbol{r}_{A}\right|^{2}+\left|\boldsymbol{r}_{B}\right|^{2}+\|\left. J\right|^{2}\right)\right]
$$

where $\|J\|^{2}=\operatorname{Tr} J^{t} J$. If $B$ is now a qubit, the state after a spin measurement along direction $\boldsymbol{k}$ on $B$ will have the form of (31) with $\frac{1}{4} \rightarrow \frac{1}{2 d_{A}}$. We then obtain, using Equation (39),

$$
S_{2}\left(\rho_{A B}^{\prime}\right)=2-\frac{1}{d_{A}}\left(\left|\boldsymbol{r}_{A}\right|^{2}+\boldsymbol{k}^{t} M_{2} \boldsymbol{k}\right)
$$

where $M_{2}=\boldsymbol{r}_{B} \boldsymbol{r}_{B}^{t}+J^{t} J$ is a $3 \times 3$ positive semidefinite symmetric matrix. Hence, $I_{2}(\boldsymbol{k})=S_{2}\left(\rho_{A B}^{\prime}\right)-$ $S_{2}\left(\rho_{A B}\right)=\frac{1}{d_{A}}\left(\operatorname{Tr} M_{2}-\boldsymbol{k}^{t} M_{2} \boldsymbol{k}\right)$. Its minimum $I_{2}(A \mid B)$ can then be evaluated analytically as [19,21]:

$$
I_{2}(A \mid B)=\operatorname{Min}_{\boldsymbol{k}} I_{2}(\boldsymbol{k})=\frac{1}{d_{A}}\left(\operatorname{Tr} M_{2}-\lambda_{1}\right)
$$

where $\lambda_{1}$ is the largest eigenvalue of $M_{2}$, the minimizing spin measurement being along the direction of the corresponding eigenvector. Equation (41) is valid for an arbitrary qudit-qubit state $\rho_{A B}$. Let us notice that the stationary condition (27) or (32) reduces, for the linear entropy, precisely to the eigenvalue equation $M_{2} \boldsymbol{k}=\lambda \boldsymbol{k}$, as in this case $f^{\prime}\left(\rho_{A B}^{\prime}\right) \propto \rho_{A B}^{\prime}$, and hence, $\alpha_{1}=\boldsymbol{r}_{B} \cdot \boldsymbol{k}, \alpha_{2}=0$ and $\alpha_{3}=1$ [21]. This indicates that the stationary measurements are those along the direction of the eigenvectors of $M_{2}$. 
For arbitrary $q>0$, we may similarly define the quantities (in what follows $c_{q}=1-2^{1-q}$ ):

$$
\begin{aligned}
I_{q}(A \mid B) & =\operatorname{Min}_{M_{B}} S_{q}\left(\rho_{A B}^{\prime}\right)-S_{q}\left(\rho_{A B}\right)=\operatorname{Min}_{M_{B}} c_{q}^{-1} \operatorname{Tr}\left(\rho_{A B}^{q}-\rho_{A B}^{\prime q}\right) \\
I_{q}^{R}(A \mid B) & =\operatorname{Min}_{M_{B}} S_{q}^{R}\left(\rho_{A B}^{\prime}\right)-S_{q}^{R}\left(\rho_{A B}\right)=\operatorname{Min}_{M_{B}} \frac{1}{1-q} \log \frac{\operatorname{Tr} \rho_{A B}^{q}}{\operatorname{Tr} \rho_{A B}^{q}} \\
& =\frac{1}{1-q} \log \left[1-\frac{c_{q} I_{q}(A \mid B)}{1-c_{q} S_{q}\left(\rho_{A B}\right)}\right]
\end{aligned}
$$

where:

$$
S_{q}^{R}(\rho)=\frac{1}{1-q} \log \operatorname{Tr} \rho^{q}=\frac{1}{1-q} \log \left[1-c_{q} S_{q}(\rho)\right], \quad q>0
$$

are the Renyi entropies [71], which are just increasing functions of the Tsallis entropies (34) (and also approach the von Neumann entropy for $q \rightarrow 1$ ). Equations (42)-(43) are again non-negative, vanishing iff $\rho_{A B}$ is of the form of (4) or (5), and approach the von Neumann information deficit (14) for $q \rightarrow 1$. Equation (44) is again just an increasing function of $I_{q}(A \mid B)$ (for fixed $\rho_{A B}$ ) and does not depend on the addition of an uncorrelated ancilla $C$ to $A\left(\rho_{A B} \rightarrow \rho_{C} \otimes \rho_{A B}\right)$, as $\operatorname{Tr} \rho_{C}^{q}$ cancels out. An analytic expression for $I_{3}(A \mid B)$ valid for any two-qubit state can also be obtained [21].

\section{Application: Quantum Correlations of Spin Pairs in XY Chains}

\subsection{Model and General Expressions}

We consider a spin $1 / 2$ system with XYZ couplings of arbitrary range, immersed in a transverse magnetic field $B$ along the $z$ axis. The Hamiltonian reads:

$$
H=B \sum_{i} s_{i z}-\frac{1}{2} \sum_{\mu=x, y, z} \sum_{i \neq j} J_{\mu}^{i j} s_{i \mu} s_{j \mu}
$$

where $s_{i \mu}$ are the (dimensionless) components of the local spin at site $i$ and $J_{\mu}^{i j}$ the coupling strengths.

The Hamiltonian (46) commutes with the $S_{z}$ spin parity operator $P_{z}$, irrespective of the coupling range, anisotropy, dimension or geometry of the system [55-57],

$$
\left[H, P_{z}\right]=0, \quad P_{z}=\exp \left[i \pi \sum_{i}\left(s_{i z}+1 / 2\right)\right]=\prod_{i}\left(-\sigma_{i z}\right)
$$

where $\sigma_{i z}=2 s_{i z}$. The non-degenerate eigenstates of $H$ will then have a definite $S_{z}$ parity $P_{z}= \pm 1$.

Consequently, the reduced density matrix of an arbitrary spin pair $i, j$ in any non-degenerate eigenstate $\left|\Psi_{\nu}\right\rangle, \rho_{i j}=\operatorname{Tr} \frac{}{(i, j)}\left|\Psi_{\nu}\right\rangle\left\langle\Psi_{\nu}\right|$, will then commute with the $S_{z}$ parity operator of the pair $P_{z}^{i j}=\sigma_{i z} \sigma_{j z}$ : $\left[\rho_{i j}, P_{z}^{i j}\right]=0$. In the standard basis $\{|00\rangle,|01\rangle,|10\rangle,|11\rangle\}, \rho_{i j}$ will therefore be an $X$-type state of the form:

$$
\rho_{i j}=\left(\begin{array}{cccc}
a_{+} & 0 & 0 & \beta \\
0 & c_{+} & \alpha & 0 \\
0 & \bar{\alpha} & c_{-} & 0 \\
\bar{\beta} & 0 & 0 & a_{-}
\end{array}\right)
$$


where the coefficients are all real (since $H$ is real in the full standard basis) and given by $\left(s_{i \pm}=s_{i x} \pm i s_{i y}\right)$ :

$$
\begin{aligned}
& a_{ \pm}=\frac{1}{4} \pm \frac{1}{2}\left\langle s_{i z}+s_{j z}\right\rangle+\left\langle s_{i z} s_{j z}\right\rangle, \\
& c_{ \pm}=\frac{1}{4} \pm \frac{1}{2}\left\langle s_{i z}-s_{j z}\right\rangle-\left\langle s_{i z} s_{j z}\right\rangle, \quad\left(\begin{array}{c}
\beta \\
\alpha
\end{array}\right)=\left\langle s_{i-} s_{j \mp}\right\rangle
\end{aligned}
$$

with $a_{+}+c_{+}+c_{-}+a_{-}=1$. This corresponds to $\boldsymbol{r}_{A}$ and $\boldsymbol{r}_{B}$ along $z$ in (29) $\left(r_{A(B)}=a_{+}+c_{+(-)}-\right.$ $c_{-(+)}-a_{-}$), with $J$ diagonal, i.e., $J_{\mu \nu}=4\left\langle s_{i \mu} s_{j \nu}\right\rangle=\delta_{\mu \nu} j_{\mu}$, with $j_{x}=2(\alpha \pm \beta), j_{z}=a_{+}+a_{-}-c_{+}-c_{-}$. The positivity of $\rho_{i j}$ implies $|\alpha| \leq \sqrt{c_{+} c_{-}},|\beta| \leq \sqrt{a_{+} a_{-}}$, with $a_{ \pm}, c_{ \pm}$non-negative. The single spin density matrix is:

$$
\rho_{i}=\operatorname{Tr}_{j} \rho_{i j}=\left(\begin{array}{cc}
a_{+}+c_{+} & 0 \\
0 & a_{-}+c_{-}
\end{array}\right)
$$

Both $\rho_{i j}$ and $\rho_{i}$ will obviously be typically mixed due to the entanglement with the rest of the chain.

In what follows, we will consider translational invariant systems, such that $\left\langle s_{i z}\right\rangle$ is site independent, i.e., $\left\langle s_{i z}\right\rangle=\left\langle s_{j z}\right\rangle \forall i, j$, implying $c_{ \pm}=c=\frac{1-a_{+}-a_{-}}{2}$. In this situation, $\rho_{i j}=\rho_{j i}$ and $D(A \mid B)=$ $D(B \mid A)=D, I_{f}(A \mid B)=I_{f}(B \mid A)=I_{f} \forall S_{f}$.

The entanglement of the pair can be measured by the entanglement of formation (6), which for two qubit states, can be evaluated as: $[65,66]$

$$
E=-\sum_{\nu= \pm} q_{\nu} \log q_{\nu}, q_{ \pm}=\frac{1}{2}\left(1 \pm \sqrt{1-C^{2}}\right)
$$

where $C$ is the concurrence $[65,66]$. For the states (48) with $c_{ \pm}=c$, the concurrence of the pair is given by:

$$
C_{i j}=2 \operatorname{Max}\left[|\beta|-c,|\alpha|-\sqrt{a_{+} a_{-}}, 0\right]
$$

The pair entanglement is of the parallel type (as in the Bell states $\frac{|00\rangle \pm|11\rangle}{\sqrt{2}}$ ) if the first entry in (53) is positive and antiparallel (as in $\frac{|01\rangle \pm|10\rangle}{\sqrt{2}}$ ) if the second entry is positive [54] (just one of them can be positive).

On the other hand, the quantum discord of the pair can be readily evaluated with the expressions (48) and (31) (see [44] for details). The ensuing minimization over the spin measurement direction $\boldsymbol{k}$ (we will consider here just projective measurements) will normally lead to the direction corresponding to maximum correlation, according to the general arguments of [72,73]. In the $X Y$ chains, which will be considered, i.e., $J_{z}^{i j}=0$, with $\left|J_{y}^{i j}\right|<J_{x}^{i j}$ and $J_{x}^{i j}>0$, the quantum discord for the states (48) will always prefer a measurement along the $x$ axis, irrespective of the field intensity [44].

The information deficit (14) can be evaluated in a similar way. In contrast with the quantum discord, the optimizing measurement direction will be affected by the field intensity, exhibiting a smooth transition from the $x$ to the $z$ direction as the field increases for the systems considered, as discussed below. The angle $\gamma$ between $\boldsymbol{k}$ and the $z$ axis can be determined from Equation (32), which leads explicitly to:

$$
\cos \gamma=\frac{\alpha_{1} r_{B}+\alpha_{2} j_{z} r_{A}}{\alpha_{3}\left(j_{x}^{2}-j_{z}^{2}\right)}
$$

when $\gamma \neq 0$ [21], which is a transcendental equation (as the $\alpha_{i}$ depend on $\gamma$ ). 
The quadratic information deficit (36) can, however, be analytically evaluated with Equation (41). Here, $M_{2}$ is already diagonal, $M_{2 \mu \nu}=\delta_{\mu \nu}\left(\delta_{\mu z} r_{B}^{2}+j_{\mu}\right)$. Assuming $\left|j_{x}\right| \geq\left|j_{y}\right|$, as will occur in the cases considered, we obtain:

$$
I_{2}=\frac{1}{2} \operatorname{Min}\left[j_{y}^{2}+j_{x}^{2}, j_{y}^{2}+r_{B}^{2}+j_{z}^{2}\right]=4 \operatorname{Min}\left[\alpha^{2}+\beta^{2}, \frac{a_{+}^{2}+a_{-}^{2}}{4}+\frac{c^{2}-\left(a_{+}-a_{-}\right) c+(\alpha-\beta)^{2}}{2}\right]
$$

with the minimizing measurement direction $\boldsymbol{k}$ along the $z(x)$ axis if the first (second) entry is minimum:

$$
\boldsymbol{k}= \begin{cases}\boldsymbol{e}_{z}, & j_{x}^{2}<r_{B}^{2}+j_{z}^{2} \\ \boldsymbol{e}_{x}, & j_{x}^{2}>r_{B}^{2}+j_{z}^{2}\end{cases}
$$

This entails that as the field $B$ increases from zero, a sharp $x \rightarrow z$ transition in the minimizing measurement direction will take place for $I_{2}$, reflecting the change in the largest eigenvalue of the matrix $M_{2}$. This transition becomes softened in the von Neumann information deficit (14), where $\boldsymbol{k}$ will evolve smoothly from the $x$ to the $z$ axis within a narrow field interval located in the vicinity of the $I_{2}$ transition. A measurement transition also occurs for other values of $q$ in the quantities (42)-(43) (see [21] for an example).

\subsection{Results}

In Figures 1 and 2, we show results for the exact ground state of a finite chain with $n$ spins coupled through cyclic $(n+1 \equiv 1)$ first neighbor anisotropic $X Y$ couplings $\left(J_{z}^{i j}=0, J_{\mu}^{i j}=\delta_{j, i \pm 1} J_{\mu}\right.$ for $\mu=x, y$ ), for which the reduced pair states (48) will depend just on the separation $L=|i-j|$ between the spins of the pair. The exact values of the elements of the density matrix (48) can be obtained, for any size $n$ or separation $L$, through the Jordan-Wigner fermionization of the model [85] and its analytic parity-dependent diagonalization $[55,86,87]$ (see the Appendix).

We will set $J_{x}>0$, with $\left|J_{y}\right| \leq J_{x}$. This involves no loss of generality as the sign of $J_{x}$ can be changed by a local rotation of angle $\pi$ around the $z$ axis at even sites (assuming $n$ even in cyclic chains), which will not affect the value of the correlation measures, and the $x$ axis can be chosen along the direction of maximum coupling.

Figure 1 depicts the behavior with increasing field $B$ of the one-way information deficits $I_{1} \equiv I$ Equation (14)) and $I_{2}$ (Equations (36)-(55)) of spin pairs in the exact definite parity ground state for the anisotropic case $J_{y}=J_{x} / 2$, together with that of the quantum discord (14) and the concurrence (53). It is first seen that $I_{1}, I_{2}$ and $D$ exhibit a similar qualitative behavior, acquiring appreciable finite values for any separation $L$ in the interval $|B|<B_{c}=\left(J_{x}+J_{y}\right) / 2$, in marked contrast with the concurrence, which is appreciable just for first and second neighbors (except for the immediate vicinity of the factorizing field; see below). The $S_{z}$ parity symmetry is essential for this result. In fact, all measures converge to a finite common value, independent of the separation $L$, at the factorizing field $[49,50,53,55-60]$ :

$$
B_{s}=\sqrt{J_{y} J_{x}}
$$

existing for $0<J_{y}<J_{x}$, where the system possesses a pair of degenerate completely separable exact ground states $[49,50,55-57]$ given by $|\Theta\rangle=|\theta, \ldots, \theta\rangle$ and $|-\Theta\rangle=P_{z}|\Theta\rangle=|-\theta, \ldots,-\theta\rangle$, where $|\theta\rangle=e^{-i \theta s_{y}}|\downarrow\rangle$ is the single spin state forming an angle $\theta$ with the $-z$ direction and $\cos \theta=B_{s} / J_{x}=$ 
$\sqrt{J_{y} / J_{x}}$. Actually, in the finite case, this field coincides with the last parity transition of the exact (and hence, of definite parity) ground state [55], such that the latter approaches, as side limits at $B=B_{s}$, the definite parity combinations [55-57]:

$$
\left|\Theta_{ \pm}\right\rangle=\frac{|\Theta\rangle \pm|-\Theta\rangle}{\sqrt{2(1 \pm\langle-\Theta \mid \Theta\rangle)}}
$$

Here, $\left|\Theta_{+}\right\rangle\left(\left|\Theta_{-}\right\rangle\right)$is the ground state limit for $B \rightarrow B_{s}^{+}\left(B \rightarrow B_{s}^{-}\right)$. Discarding the overlap $\langle-\Theta \mid \Theta\rangle=\cos ^{n} \theta$, which is negligible if $n$ and $\theta$ are not too small $\left(\cos ^{n} \theta \approx e^{-n \theta^{2} / 2}\right.$ for small $\left.\theta\right)$, Equation (58) leads to a common reduced state for any pair $i, j$, given by [44,55]:

$$
\rho_{\theta}=\frac{1}{2}(|\theta\rangle\langle\theta|\otimes| \theta\rangle\langle\theta|+|-\theta\rangle\langle-\theta|\otimes|-\theta\rangle\langle-\theta|)
$$

This is a separable mixed state, and therefore, it leads to a zero concurrence for any pair, as seen in Figure 1 (where results at $B_{s}$ correspond to the side limits (58)). However, it is not of the classically-correlated form of (4) or (5) if $\langle-\theta \mid \theta\rangle=\cos \theta \neq 0$ or 1, i.e., if $| \pm \theta\rangle$ are non-orthogonal and distinct, leading then to a common appreciable value of $D, I_{1}, I_{2}$ and, in fact, all $I_{f}$. We also notice that the same reduced state (59) is obtained from the mixture $\frac{1}{2}(|\Theta\rangle\langle\Theta|+|-\Theta\rangle\langle-\Theta|)$, which represents the low temperature limit of the thermal state $\rho \propto \exp [-H / k T]$ at $B=B_{s}$.

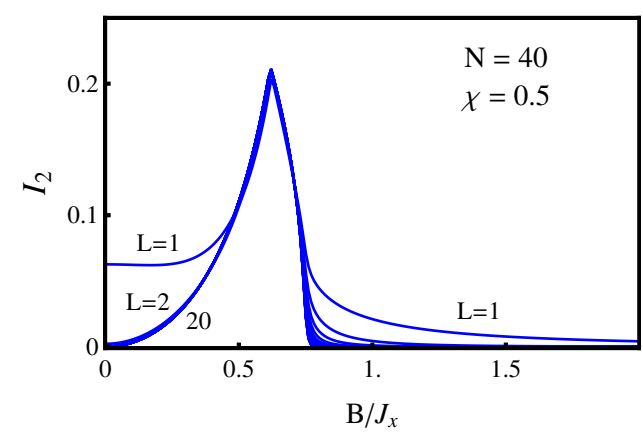

(a)

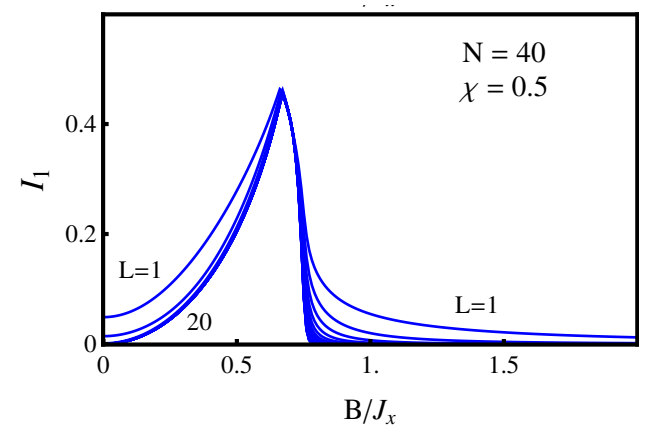

(c)

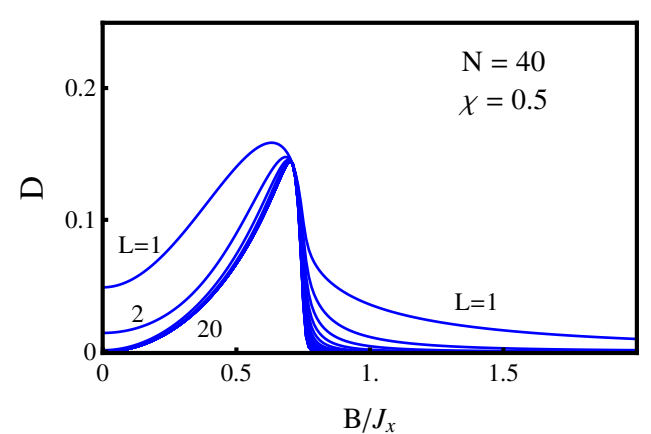

(b)

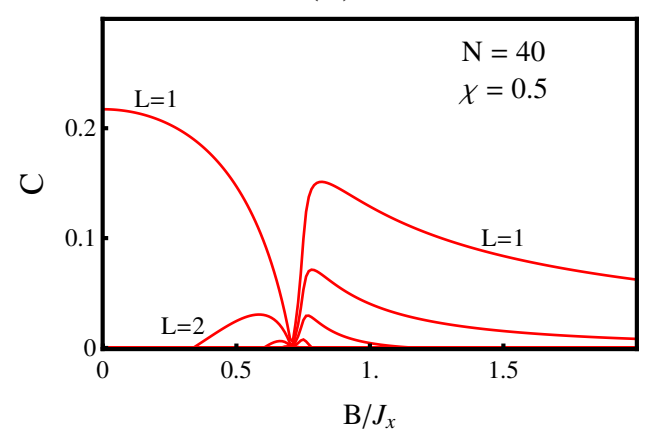

(d)

Figure 1. (a,c) The one-way information deficits $I_{2}$, Equation (36) (a) and $I_{1}$, Equation (14) (c) as a function of the scaled magnetic field $B / J_{x}$, for spin pairs with separation $L=1,2, \ldots, n / 2$ in the exact ground state of a cyclic chain of $n=40$ spins with first neighbor anisotropic $X Y$ couplings $\left(\chi=J_{y} / J_{x}=1 / 2\right)$. (b,d) The quantum discord $D$, Equation (10) (b) and the concurrence $C$, Equation (53) (d) for the same pairs. The results for different separations coincide exactly at the factorization field $B_{s}=\sqrt{J_{y} J_{x}} \approx 0.71 J_{x}$. 

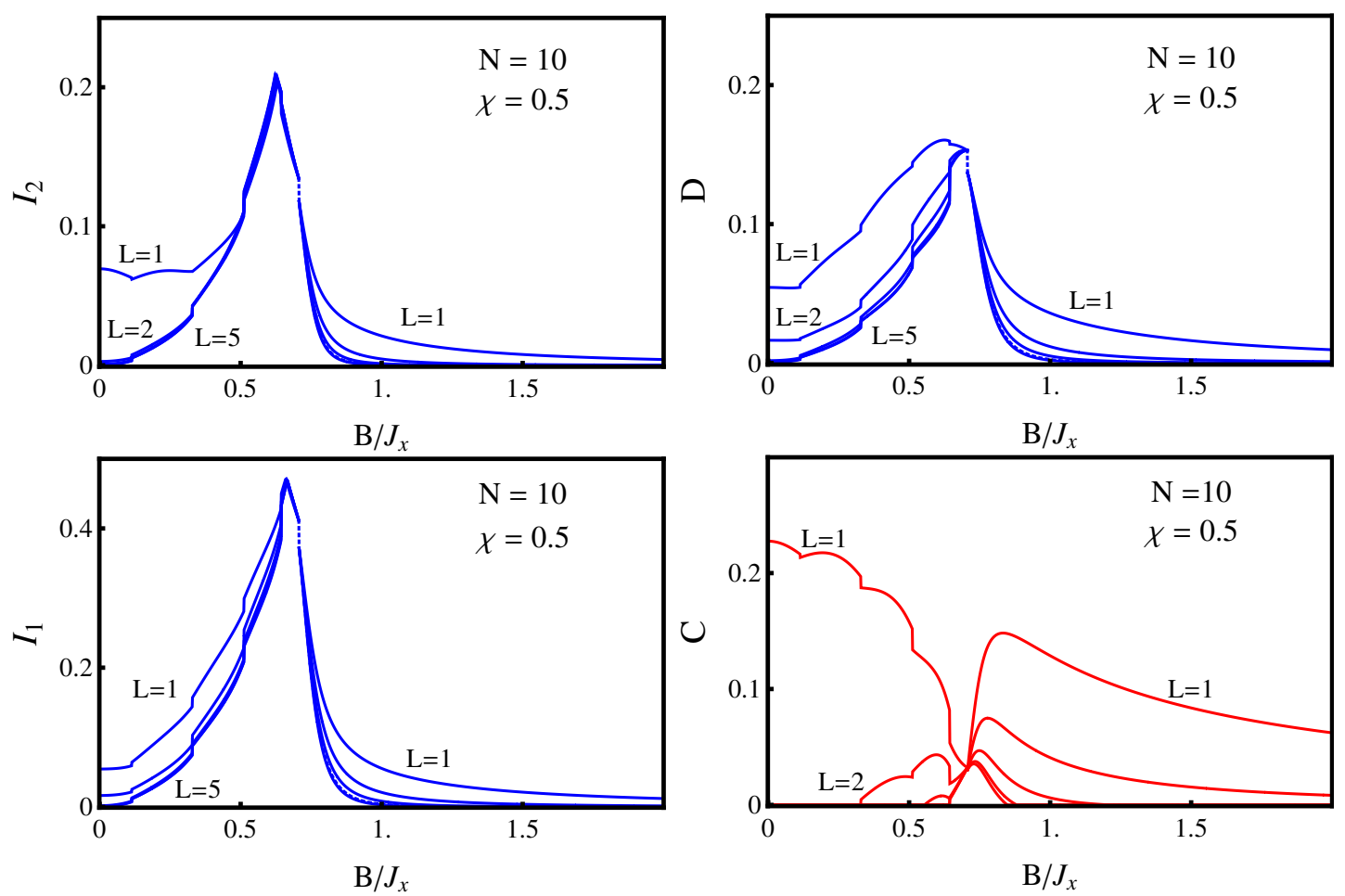

Figure 2. The same quantities of Figure 1 for $n=10$ spins. In this case, the parity transitions of the ground state lead to small, but appreciable discontinuities in all quantities, with the last transition (indicated by the vertical dotted line) taking place at the factorizing field $B_{s}$. For this size, the concurrence also presents small, but finite side limits at $B_{s}$.

It is then possible to obtain straightforward analytic expressions for the side limits of $D$ [44], $I_{2}$ and $I_{1}$ at the factorizing field through the state (59), which leads to $a_{ \pm}=\frac{1}{4}(1 \pm \cos \theta)^{2}$ and $\alpha=\beta=c=\frac{1}{4} \sin ^{2} \theta$ in (48), with $\cos ^{2} \theta=J_{y} / J_{x}$. That for $I_{2}$ is particularly clean and given by:

$$
I_{2}\left(B_{s}\right)=\left\{\begin{array}{ll}
\frac{(1-\chi)^{2}}{2}, & \chi \geq 1 / 3 \\
\frac{\chi(1+\chi)}{2}, & \chi \leq 1 / 3
\end{array} \quad \text { where } \chi=J_{y} / J_{x}\right.
$$

with the minimizing measurement at $B_{s}$ being along $z$ if $\chi>1 / 3$ and along $x$ if $\chi<1 / 3$. Equation (60) applies for all separations $L$.

For small chains, the results are similar, but the effects of the parity transitions of the ground state (it undergoes $n / 2$ parity transitions as the field increases from zero, the last one at $B=B_{s}$ [55]) are now appreciable trough the finite discontinuities exhibited by $I_{2}, I_{1}$ and $D$, as seen in Figure 2 . At the factorizing field, these discontinuities arise from the overlap $\langle-\Theta \mid \Theta\rangle$, which now cannot be strictly neglected. This leads to an additional term $\propto \pm \cos ^{n-2} \theta(|\theta\rangle\langle-\theta|\otimes| \theta\rangle\langle-\theta|+$ h.c. $)$ in Equation (59), which originates slightly distinct side limits of $D$ [44] and also $I_{2}$ and $I_{1}$ at $B_{s}$. Moreover, it also leads to small, but finite and distinct common side limits of the concurrence at $B=B_{s}$ [55-57], which was known to reach full range in its vicinity [54]. All of these side limits are, nevertheless, still independent of the pair separation $L$. In the case of $I_{2}$, they are given, for $\chi \gtrsim 1 / 3$, by:

$$
I_{2}\left(B_{s}^{ \pm}\right)=\frac{(1-\chi)^{2}}{2} \frac{1+\chi^{n-2}}{\left(1 \pm \chi^{n / 2}\right)^{2}}
$$


which corrects the upper line in Equation (60) for finite $n$ (or $\chi \rightarrow 1$ ), and $+(-$ ) corresponds to the right (left) side limit. The side limits of the concurrence are $C\left(B_{s}^{ \pm}\right)=\frac{\chi^{n / 2-1}(1-\chi)}{1 \pm \chi^{n / 2}}$, as obtained from (53) [55-57].

The behavior of the quantum discord for longer range ferromagnetic-type couplings is qualitatively similar [44]. Moreover, a factorizing field still exists for longer range couplings with a constant anisotropy $\chi=J_{y}^{i j} / J_{x}^{i j}$ [56,57], in which case the reduced pair state at $B_{s}$ is again given by Equation (59) with $\cos \theta=\sqrt{\chi}$, and Equations (60)-(61) remain then valid.

In Figure 3, we compare the behavior of $I_{2}, I_{1}$ and $D$ for first neighbors in the chains of Figures 1 and 2, with that of the associated entanglement monotone, i.e., the squared concurrence $C^{2}$ for $I_{2}$ and the entanglement of formation $E$ for $I_{1}$ and $D$, such that both quantities coincide for pure states. It is seen that for strong fields, differences are very small, in agreement with the weak entanglement of the pair with the rest of the chain in this regime $\left(\rho_{i, i+1}\right.$ is almost pure). The strong differences arise for $B<B_{c}$ and especially in the vicinity of the factorizing field, due to the arguments exposed above. For $|B|<B_{c}$, the reduced pair state becomes appreciably mixed in the definite parity ground states, including the states (58) at the factorizing field, due to the entanglement with the rest of the chain. Significant differences between $I_{f}$ (and $D$ ) with the corresponding entanglement monotone become then feasible.
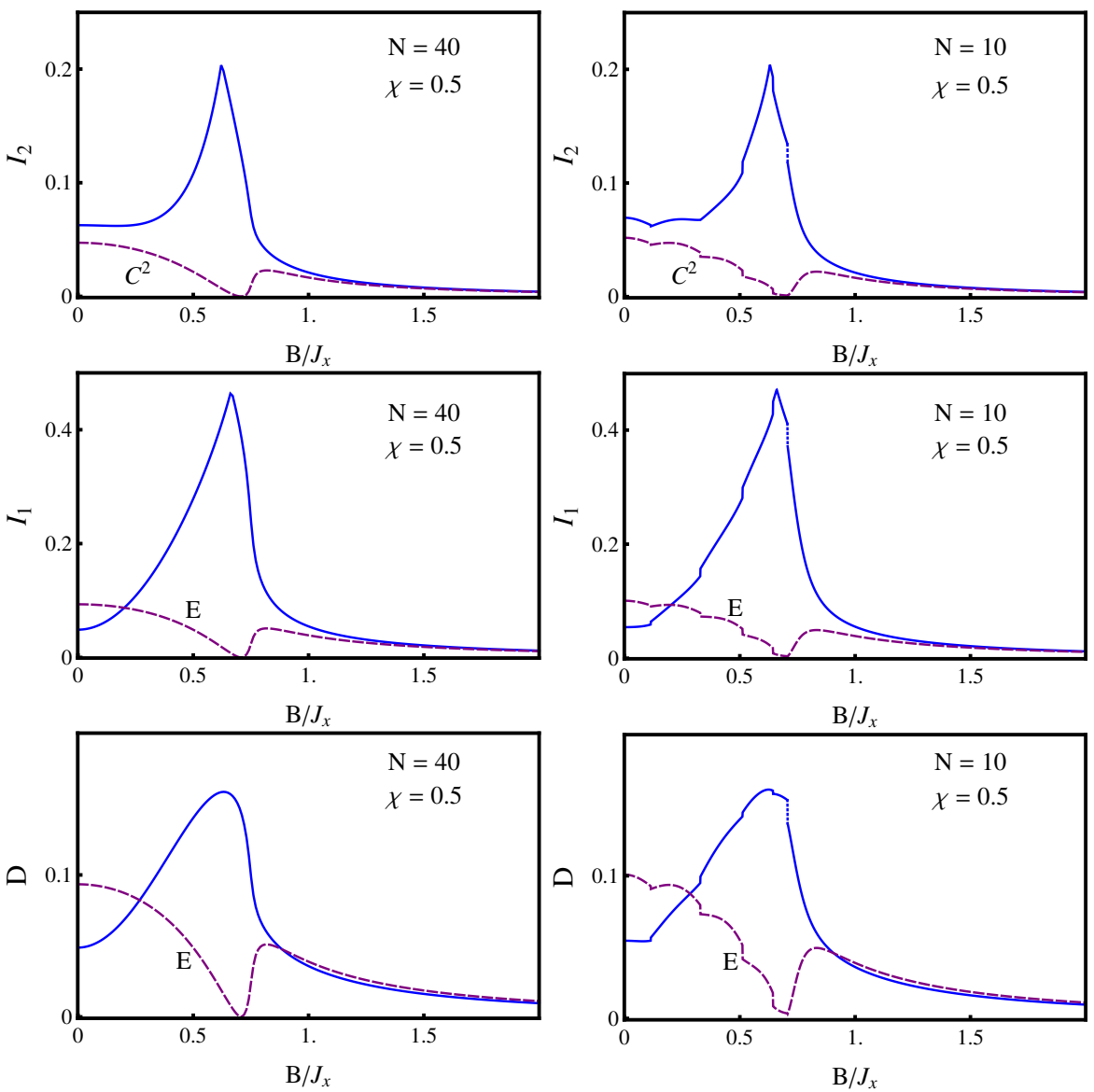

Figure 3. Plot of $I_{2}$ (top), $I_{1}$ (center) and $D$ (bottom) together with the associated entanglement monotones for a first neighbor pair $(L=1)$ in the ground state of the chains of Figures 1 and 2. 
It is also seen that $I_{2}$ is in this case an upper bound of $C^{2}$ for all fields, whereas $I_{1}$ is not an upper bound of $E$ for low fields, while $D$ is not an upper bound, even for strong fields, indicating the lack of an order relationship between $D$ and $E$, even in this regime. In the case of $I_{2}$, it is easy to show from Equations (53) and (55) that for $X$ states, it is always an upper bound of $C^{2}$ when the minimizing measurement is along $z$ [21]. In, fact, for strong fields $|B| \gg J_{x}$, a perturbative expansion [20] for the present chain leads to $C \approx 2\left(\eta-\eta^{2}\right), I_{2} \approx 4 \eta^{2}, I_{1} \approx \eta^{2}\left(\log e-\log \eta^{2}\right)$ and $D \approx$ $\eta^{2}\left(\log e-\log \eta^{2}-2\right)$, where:

$$
\eta=\frac{J_{x}-J_{y}}{8 B}
$$

Hence, in this limit, $I_{2}-C^{2}=O\left(\eta^{3}\right)$ and $I_{1}-E=O\left(-\eta^{3} \log \eta^{2}\right)$, both positive, whereas $D-E \approx$ $O\left(-\eta^{2}\right)$ becomes negative.

\subsection{Minimizing Measurement}

Although $I_{1}, I_{2}$ and $D$ show a similar qualitative behavior, both measures $I_{1}$ and $I_{2}$ exhibit a more pronounced maximum, in comparison to that of the quantum discord, as appreciated in Figures 1-3. This reflects the transition in the orientation of their local minimizing spin measurements as the field increases, which, as mentioned above, is not present in the quantum discord. The latter prefers in the present system a measurement along the $x$ axis, even for large fields and for any separation between the spins, following the strongest correlation [72,73]. As seen in Figure 4 and as previously stated, $I_{2}$ exhibits instead a sharp transition from a direction parallel to the $x$ axis $(\gamma=\pi / 2)$ to a direction parallel to the $z$ axis $(\gamma=0)$, i.e., parallel to the field. This transition takes place, in the case shown in Figure 1 , for all separations $L$ at $B \approx 0.65 J_{x}$. In the case of the information deficit $I_{1}$, the transition becomes smooth, as the angle $\gamma$ takes all of the intermediate values between zero and $\pi / 2$ (as determined by Equation (54)) for all separations in a narrow field interval centered at the $I_{2}$ critical field, as also seen in Figure 4.

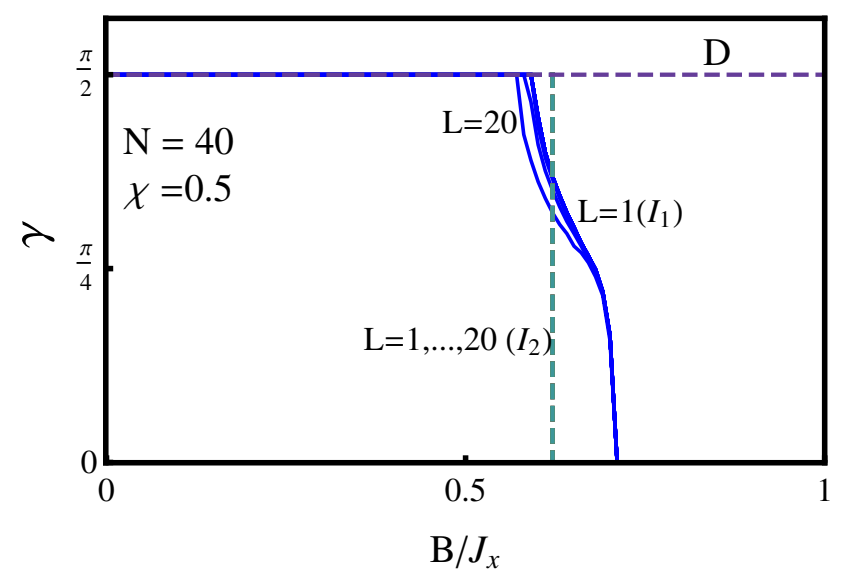

Figure 4. The angle $\gamma$ determining the direction of the minimizing local spin measurement for $D, I_{1}$ and $I_{2}$, as a function of the scaled transverse magnetic field, for a chain of $n=40$ spins with $J_{y}=J_{x} / 2$. Results for all separations $L$ of the pair are shown.

The value of the field where the transition in the optimizing local measurement for $I_{2}$ occurs depends on the anisotropy, but only slightly on the separation $L$, except in the $X X$ limit $\left(J_{y} \rightarrow J_{x}\right)$, as can be 
seen in the left panel of Figure 5. The same holds for the field interval where the "transition" (actually the evolution from $\pi / 2$ to zero of the measurement angle $\gamma$ ) in $I_{1}$ takes place (Figure $5 \mathrm{~b}$ ). In the case of $I_{2}$, if $\chi=1 / 3$, the measurement transition for all separations $L$ occur exactly at the factorizing field $B_{s}=\sqrt{\chi} J_{x}$, as follows from Equation (60).

The measurement transition reflects essentially the qualitative change experienced by the reduced state of the pair for increasing fields. Away from the $X X$ limit, the dominant eigenstate of $\rho_{i j}$ (that with the largest eigenvalue) for not too low fields is the entangled state $\left|\Psi_{+}\right\rangle=u|\downarrow \downarrow\rangle+v|\uparrow \uparrow\rangle$ with $v / u=$ $\frac{\beta}{\varepsilon+\sqrt{\varepsilon^{2}+\beta^{2}}}$ and $\varepsilon=\frac{a_{-}-a_{+}}{2}$. Above the measurement transition field (i.e., when the optimum measurement is parallel to the field), $v / u$ becomes small $(\lesssim 0.25)$, indicating that the pair is approximately aligned with the field. Instead, below the transition field, $v / u$ increases, approaching one for $B \rightarrow 0$ (where $\left|\Psi_{+}\right\rangle$becomes a parallel Bell state), and the least disturbing measurement is along $x$. For very low fields, the dominant eigenstate may shift to the antiparallel Bell state $\left|\Psi_{-}\right\rangle=\frac{|\uparrow \downarrow\rangle+|\downarrow \uparrow\rangle}{\sqrt{2}}$ arising from the central block of (48), and in this case, the measurement along $x$ is still preferred. On the other hand, in the $X X$ limit, $\beta=0$ in (48), and the dominant eigenstate is either $\left|\Psi_{-}\right\rangle$at low fields or $\left|\Psi_{+}\right\rangle=|\downarrow \downarrow\rangle$ for strong fields; and the measurement transition of $I_{2}$ indicates essentially the field where the sharp transition in the dominant eigenstate (from maximally entangled to separable) takes place [51]. Such a measurement transition for increasing fields persists even at finite temperatures [51].

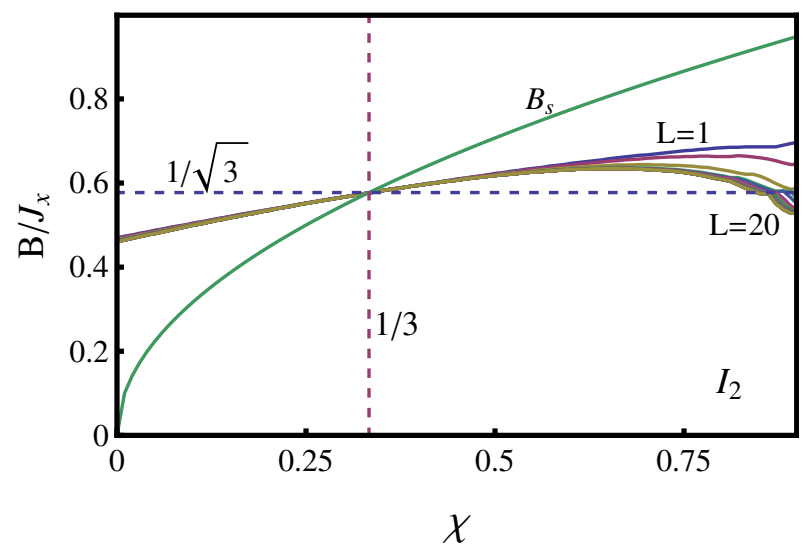

(a)

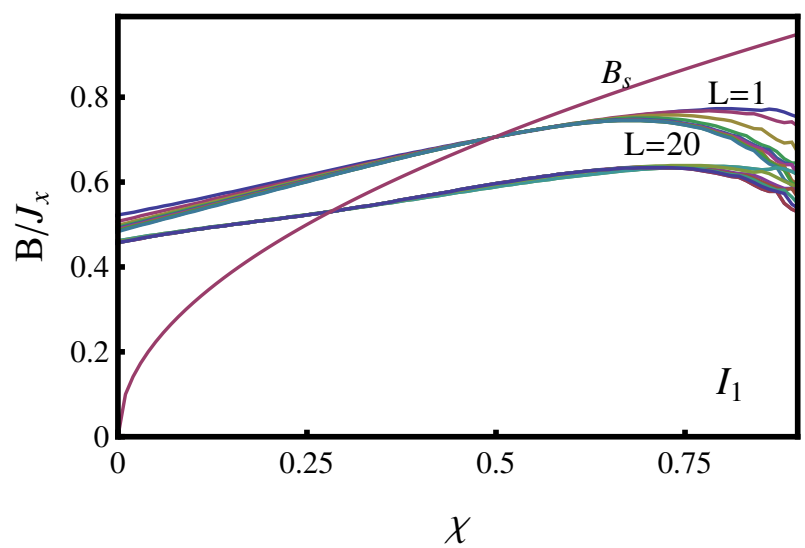

(b)

Figure 5. (a) The field where the transition in the minimizing measurement of $I_{2}$ takes place, as a function of the anisotropy $\chi=J_{y} / J_{x}$. A direction along the $x(z)$ axis is preferred below (above) the transition field. The factorizing field $B_{s}$ is also shown. All transition fields coincide with the factorizing field if $\chi=1 / 3$, Equation (60). (b) The fields delimiting the interval where the smoothed transition in the minimizing measurement of the von Neumann information deficit $I_{1}$ takes place.

\section{Conclusions}

We have examined the behavior of the quantum discord and the standard and quadratic one-way information deficit of spin pairs in the exact definite parity ground state of a finite anisotropic cyclic $X Y$ spin $1 / 2$ chain in a transverse field. We have first provided a brief overview of the quantum discord, the standard von Neumann-based one-way information deficit and the generalized information deficit, which 
contains the standard, as well as the quadratic deficit as particular cases and which can be interpreted as a measure of the minimum entanglement generated between the system and the measurement apparatus after a complete local projective measurement. The first important result is that the behavior of all of these measures is quite distinct from that of the pair entanglement for fields below the critical field, acquiring finite appreciable values for all separations of the spins of the pair. Moreover, they reach (as side limits) a common (independent of the separation) finite value at the factorizing field, which in a finite chain, is the field where the last ground state parity transition takes place. These finite limits can be evaluated analytically. The entanglement of pairs also reaches full range in its vicinity, although its value is much smaller and vanishes at this field, except for very small samples. Parity effects are of crucial importance for the proper description of these measures in finite systems below the critical field.

The second important result is that the behavior of the optimizing local spin measurement of both the standard and generalized information deficit is quite distinct from that optimizing the quantum discord, exhibiting a transition in the direction of the spin measurement, from that of maximum correlation to that parallel to the field. The details of this transition depend on the choice of entropy (it is sharp for $I_{2}$ and smooth for $I_{1}$ ). The quantum discord prefers instead that of maximum correlation, even for strong fields. Hence, the quantum discord, which is based on the minimization of a conditional entropy, "detects" in this way this direction $[72,73]$, while the information deficits, based on the minimization of a total entropy, are more sensible to changes in the structure of the reduced state of the pair.

A final comment is that the generalized formalism permits the use of simple entropic forms involving just low powers of the density matrix, leading to measures of the form of (42) or (43), which can be more easily evaluated and optimized and which are also more easily accessible from the experimental side.

\section{A. Appendix}

We briefly discuss here the exact solution of the finite cyclic $X Y$ chain with first neighbor couplings, which requires one to take into account exactly the parity effects [55,85-87]. The Jordan-Wigner transformation [85] allows one to rewrite the Hamiltonian (46) in the $X Y$ case $\left(J_{z}^{i j}=0\right)$ for $J_{\mu}^{i j}=$ $J_{\mu} \delta_{i, j \pm 1}, \mu=x, y$, and for each value \pm 1 of the $S_{z}$ parity $P_{z}$, as a quadratic form in fermion creation and annihilation operators $c_{i}^{\dagger}, c_{i}$ defined by $c_{i}^{\dagger}=s_{i+} \exp \left[-i \pi \sum_{j=1}^{i-1} s_{j+} s_{j-}\right]$, with the reverse transformation given by $s_{i+}=c_{i}^{\dagger} \exp \left[i \pi \sum_{j=1}^{i-1} c_{j}^{\dagger} c_{j}\right]$. This leads to:

$$
\begin{aligned}
H^{ \pm} & =\sum_{i=1}^{n} B\left(c_{i}^{\dagger} c_{i}-\frac{1}{2}\right)-\frac{1}{2} \eta_{i}^{ \pm}\left(J_{+} c_{i}^{\dagger} c_{i+1}+J_{-} c_{i}^{\dagger} c_{i+1}^{\dagger}+\text { h.c. }\right) \\
& =\sum_{k \in K_{ \pm}} \lambda_{k}\left(a_{k}^{\dagger} a_{k}-\frac{1}{2}\right)
\end{aligned}
$$

where $J_{ \pm}=\frac{1}{2}\left(J_{x} \pm J_{y}\right)$ and $n+1 \equiv 1, \eta_{i}^{-}=1, \eta_{i}^{+}=1-2 \delta_{\text {in }}$ [85]. In (62), $K_{+}=\left\{\frac{1}{2}, \ldots, n-\frac{1}{2}\right\}$, $K_{-}=\{0, \ldots, n-1\}$ and:

$$
\lambda_{k}=\sqrt{\left(B-J_{+} \cos \omega_{k}\right)^{2}+J_{-}^{2} \sin ^{2} \omega_{k}}, \quad \omega_{k}=2 \pi k / n
$$


The last form of (62) is obtained through a parity-dependent discrete Fourier transform $c_{j}^{\dagger}=$ $\frac{e^{i \pi / 4}}{\sqrt{n}} \sum_{k \in K_{ \pm}} e^{-i \omega_{k} j} c_{k}^{\prime \dagger}$, followed by a BCS-type Bogoliubov transformation $c_{k}^{\prime \dagger}=u_{k} a_{k}^{\dagger}+v_{k} a_{n-k}, c_{n-k}^{\prime}=$ $u_{k} a_{n-k}-v_{k} a_{k}^{\dagger}$ to quasiparticle fermionic operators $a_{k}, a_{k}^{\dagger}$, with $\left(\begin{array}{c}u_{k}^{2} \\ v_{k}^{2}\end{array}\right)=\frac{1}{2}\left[1 \pm\left(B-J_{+} \cos \omega_{k}\right) / \lambda_{k}\right]$.

For $B \geq 0$, we may set $\lambda_{k} \geq 0$ for $k \neq 0$ and $\lambda_{0}=J_{+}-B$, in which case, the quasiparticle vacuum of $H^{ \pm}$has the right parity, and the lowest energy is $E^{ \pm}=-\frac{1}{2} \sum_{k \in K_{ \pm}} \lambda_{k}$. At the factorizing field (57), $\lambda_{k}=J_{+}-B_{s} \cos \omega_{k}$ and $E^{ \pm}=-n J_{+} / 2$ [55].

The reduced state of a spin pair in the exact ground state can then be obtained from the basic contractions $\left\langle a_{k}^{\dagger} a_{k^{\prime}}\right\rangle=0,\left\langle a_{k}^{\dagger} a_{k^{\prime}}^{\dagger}\right\rangle=0$, leading to $\left\langle c^{\prime \dagger} c^{\prime} k^{\prime}\right\rangle=v_{k}^{2} \delta_{k k^{\prime}},\left\langle{c^{\prime}}_{k}^{\dagger} c^{\prime \dagger}{ }^{\prime}\right\rangle=u_{k} v_{k} \delta_{k,-k^{\prime}}$ and $(L=i-j)$ :

$$
\left\langle c_{i}^{\dagger} c_{j}\right\rangle_{ \pm}=\frac{1}{n} \sum_{k \in K_{ \pm}} e^{-i \omega_{k} L} v_{k}^{2}=f_{L}+\frac{1}{2} \delta_{i j}, \quad\left\langle c_{i}^{\dagger} c_{j}^{\dagger}\right\rangle_{ \pm}=\frac{1}{n} \sum_{k \in K_{ \pm}} e^{-i \omega_{k} L} u_{k} v_{k}=g_{L}
$$

The application of Wick's theorem then leads to $[44,85]$ :

$$
\begin{aligned}
\left\langle s_{i z}\right\rangle & =f_{0},\left\langle s_{i z} s_{j z}\right\rangle=f_{0}^{2}-f_{L}^{2}+g_{L}^{2} \\
\left\langle s_{i-} s_{j \mp}\right\rangle & =\frac{1}{4}\left[\operatorname{det}\left(A_{L}^{+}\right) \mp \operatorname{det}\left(A_{L}^{-}\right)\right]
\end{aligned}
$$

where $\left(A_{L}^{ \pm}\right)$are $L \times L$ matrices of elements $\left(A_{L}^{ \pm}\right)_{i j}=2\left(f_{i-j \pm 1}+g_{i-j \pm 1}\right)$. These results, valid for any finite $n$, were checked through direct diagonalization for small $n$.

\section{Acknowledgments}

Norma Canosa and Leonardo Ciliberti acknowledge support from CONICET and Raúl Rossignoli acknowledges the support from CIC of Argentina.

\section{Author Contributions}

All authors contributed equally to this paper. All authors have read and approved the final manuscript.

\section{Conflicts of Interest}

The authors declare no conflict of interest.

\section{References}

1. Schrödinger, E. Die gegenwärtige situation in der Quantenmechanik. Naturwissenschaften 1935, 23, 807-812.

2. Schrödinger, E. Discussion of probability relations between separated systems. Proc. Cambridge Philos. Soc. 1935, 31, 555-563.

3. Schumacher, B. Quantum coding. Phys. Rev. A 1995, 51, 2738-2747.

4. Bennett, C.H.; Bernstein, H.J.; Popescu, S.; Schumacher, B. Concentrating partial entanglement by local operations. Phys. Rev. A 1996, 53, 2046-2052.

5. Nielsen, M.A.; Chuang, I. Quantum Computation and Quantum Information; Cambridge University Press: London, UK, 2000; pp. 1-676. 
6. Vedral, V. Introduction to Quantum Information Science; Oxford University Press: Oxford, UK, 2006; pp. 1-183.

7. Haroche S.; Raimond, J.M. Exploring the Quantum; Oxford University Press: Oxford, UK, 2007; pp. 1-605.

8. Werner, R.F. Quantum states with Einstein-Podolsky-Rosen correlations admitting a hidden-variable model. Phys. Rev. A 1989, 40, 4277-4281.

9. Ollivier, H.; Zurek, W.H. Quantum Discord: A Measure of the Quantumness of Correlations. Phys. Rev. Lett. 2001, 88, 017901.

10. Henderson, L.; Vedral, V. Classical, quantum and total correlations. J. Phys. A 2001, 34, 6899-6905.

11. Vedral, V. Classical Correlations and Entanglement in Quantum Measurements. Phys. Rev. Lett. 2003, 90, 050401.

12. Zurek, W.H. Decoherence, einselection, and the quantum origins of the classical. Rev. Mod. Phys. 2003, 75, 715-775.

13. Zurek, W.H. Quantum discord and Maxwells demons. Phys. Rev. A 2003, 67, 012320.

14. Modi, K.; Paterek, T.; Son, W.; Vedral, V.; Williamson, M. Unified View of Quantum and Classical Correlations. Phys. Rev. Lett. 2010 104, 080501.

15. Modi, K.; Brodutch, A.; Cable, H.; Paterek, T.; Vedral, V. The classical-quantum boundary for correlations: Discord and related measures. Rev. Mod. Phys. 2012, 84, 1655-1707.

16. Horodecki, M.; Horodecki, P.; Horodecki, R.; Oppenheim, J.; Sen De, A.; Sen, U.; Synak, B. Local versus nonlocal information in quantum-information theory: Formalism and phenomena. Phys. Rev. A 2005, 71, 062307.

17. Oppenheim, J.; Horodecki, M.; Horodecki, P.; Horodecki, R. Thermodynamical Approach to Quantifying Quantum Correlations. Phys. Rev. Lett. 2002, 89, 180402.

18. Streltsov, A.; Kampermann, H.; Bruß, D. Linking Quantum Discord to Entanglement in a Measurement. Phys. Rev. Lett. 2011, 106, 160401.

19. Dakić, B.; Vedral, V.; Brukner, Č. Necessary and Sufficient Condition for Nonzero Quantum Discord. Phys. Rev. Lett. 2010, 105, 190502.

20. Rossignoli, R.; Canosa, N.; Ciliberti, L. Generalized entropic measures of quantum correlations. Phys. Rev. A 2010, 82, 052382.

21. Rossignoli, R.; Canosa, N.; Ciliberti, L. Quantum correlations and least disturbing local measurements. Phys. Rev. A 2011, 84, 052329.

22. Girolami, D.; Tufarelli, T.; Adesso, G. Characterizing Nonclassical Correlations via Local Quantum Uncertainty. Phys. Rev. Lett. 2013, 110, 240402.

23. Luo, S.; Fu, S.; Oh, C.H. Quantifying correlations via the Wigner-Yanase skew information. Phys. Rev. A 2012, 85, 032117.

24. Paula, M.; de Oliveira, T.R.; Sarandy, M.S. Geometric quantum discord through the Schatten 1-norm. Phys. Rev. A 2013, 87, 064101.

25. Hu, H.; Fan, H.; Zhou, D.L.; Liu, W.M. Quantum correlating power of local quantum channels. Phys. Rev. A 2013, 87, 032340. 
26. Nakano, T.; Piani, M.; Adesso, G. Negativity of quantumness and its interpretations. Phys. Rev. A 2013, 88, 012117.

27. Ciccarello, F.; Tuffarelli, T.; Giovannetti, V. Toward computability of trace distance discord. New J. Phys. 2014, 16, 013038.

28. Bennett, C.H., Brassard, G.; Crépeau, C.; Jozsa, R.; Peres, A.; Wootters, W.K. Teleporting an unknown quantum state via dual classical and Einstein-Podolsky-Rosen channels. Phys. Rev. Lett. 1993, 70, 1895-1899.

29. Josza, R.; Linden, N. On the role of entanglement in quantum computational speed-up. Proc. $R$. Soc. 2003, A459, 2011-2032.

30. Vidal, G. Efficient Classical Simulation of Slightly Entangled Quantum Computations. Phys. Rev. Lett. 2003, 91, 147902.

31. Knill, E.; Laflamme, R. Power of One Bit of Quantum Information. Phys. Rev. Lett. 1998, 81, 5672-5675.

32. Datta, A.; Flammia, S.T.; Caves, C.M. Entanglement and the power of one qubit. Phys. Rev. A 2005, 72, 042316.

33. Datta, A.; Shaji, A.; Caves, C.M. Quantum Discord and the Power of One Qubit. Phys. Rev. Lett. 2008, 100, 050502.

34. Piani, M.; Gharibian, S.; Adesso, G.; Calsamiglia, J.; Horodecki, P.; Winter, A. All nonclassical correlations can be activated into distillable entanglement. Phys. Rev. Lett. 2011, 106, 220403.

35. Koashi, M.; Winter, A. Monogamy of quantum entanglement and other correlations. Phys. Rev. A 2004, 69, 022309.

36. Madhok, V.; Datta, A. Interpreting quantum discord through quantum state merging. Phys. Rev. A 2011, 83, 032323.

37. Cavalcanti, D.; Aolita, L.; Boixo, S.; Modi, K.; Piani, M.; Winter, A. Operational interpretations of quantum discord. Phys. Rev. A 2011, 83, 032324.

38. Fanchini, F.F.; Cornelio, M.F.; de Oliveira, M.C.; Caldeira, A.O. Conservation law for distributed entanglement of formation and quantum discord. Phys. Rev. A 2011, 84, 012313.

39. Adesso, G.; D’Ambrosio, V.; Nagali, E.; Piani, M.; Sciarrino, F. Experimental Entanglement Activation from Discord in a Programmable Quantum Measurement. Phys. Rev. Lett. 2014, 112, 140501.

40. Huang, I. Computing quantum discord is NP complete. New. J. Phys. 2014, 16, 033027.

41. Dillenschneider, R. Quantum discord and quantum phase transition in spin chains. Phys. Rev. $B$ 2008, 78, 224413.

42. Maziero, J.; Guzman, H.C.; Céleri, L.C.; Sarandy, M.S.; Serra, R.M. Quantum and classical thermal correlations in the XY spin-1/2 chain. Phys. Rev. A 2010, 82, 012106.

43. Werlang, T. ; Rigolin, G. Thermal and magnetic quantum discord in Heisenberg models. Phys. Rev. A 2010, 81, 044101.

44. Ciliberti, L.; Rossignoli, R.; Canosa, N. Quantum discord in finite $X Y$ chains. Phys. Rev. A 2010, $82,042316$.

45. Werlang, T.; Ribeiro, G.A.P.; Rigolin, G. Spotlighting quantum critical points via quantum correlations at finite temperatures. Phys. Rev. A 2011, 83, 062334. 
46. Liu, B.-Q.; Shao, B.; Li, J.-G.; Zou, J.; Wu, L.-A. Quantum and classical correlations in the one-dimensional XY model with Dzyaloshinskii-Moriya interaction. Phys. Rev. A 2011, 83, 052112.

47. Li, Y.-C.; Lin, H.-Q. Thermal quantum and classical correlations and entanglement in the XY spin model with three-spin interaction. Phys. Rev. A 2011, 83, 052323.

48. Rossignoli, R.; Canosa, N; Matera, J.M. Measurements, quantum discord, and parity in spin-1 systems. Phys. Rev. A 2012, 86, 022104.

49. Campbell, S.; Mazzola, L.; de Chiara, G.; Apollaro, T.J.G.; Plastina, F.; Busch, T.; Paternostro, M. Global quantum correlations in finite-size spin chains. New J. Phys. 2013, 15, 043033.

50. Campbell, S.; Richens, J.; Lo Gullo, N.; Busch, T. Criticality, factorization, and long-range correlations in the anisotropic XY model. Phys. Rev. A 2013, 88, 062305.

51. Ciliberti, L.; Canosa, N.; Rossignoli, R. Discord and Information Deficit in $X X$ chains. Phys. Rev. A 2013, 88, 012119.

52. Huang, Y.; Scaling of quantum discord in spin models. Phys. Rev. B 2014, 89, 054410.

53. Kurmann, J.; Thomas, H.; Müller, G. Antiferromagnetic long-range order in the anisotropic quantum spin chain. Physica A 1982, 112, 235-255.

54. Amico, L.; Baroni, F.; Fubini, A.; Patane, D.; Tognetti, V.; Verrucchi, P. Divergence of the entanglement range in low-dimensional quantum systems. Phys. Rev. A 2006, 74, 022322.

55. Rossignoli, R.; Canosa, N.; Matera, J.M. Entanglement of finite cyclic chains at factorizing fields. Phys. Rev. A 2008, 77, 052322.

56. Rossignoli, R.; Canosa, N.; Matera, J.M. Factorization and entanglement in general xyz spin arrays in nonuniform transverse fields. Phys. Rev. A 2009 80, 062325.

57. Canosa, N.; Rossignoli, R.; Matera, J.M. Separability and entanglement in finite dimer-type chains in general transverse fields. Phys. Rev. B 2010, 81, 054415.

58. Giampaolo, S.M.; Adesso, G.; Illuminati, F. Theory of ground state factorization in quantum cooperative systems. Phys. Rev. Lett. 2008, 100, 197201.

59. Giampaolo, S.M.; Adesso, G.; Illuminati, F. Separability and ground-state factorization in quantum spin systems. Phys. Rev. B 2009, 79, 224434.

60. Giampaolo, S.M.; Adesso, G.; Illuminati, F. Probing quantum frustrated systems via factorization of the ground state. Phys. Rev. Lett. 2010, 104, 207202.

61. Peres, A. Separability criterion for density matrices. Phys. Rev. Lett. 1996, 77, 1413-1415.

62. Horodecki, M.; Horodecki, P.; Horodecki, R. Separability of mixed states: Necessary and sufficient conditions. Phys. Lett. A 1996, 223, 1-8.

63. Bennett, C.H.; DiVincenzo, D.P.; Smolin, J.A.; Wootters, W.K. Mixed-state entanglement and quantum error correction. Phys. Rev. A 1996, 54, 3824-3851.

64. Vidal, G. Entanglement monotones. J. Mod. Opt. 2000, 47, 355-376.

65. Hill, S.; Wootters, W.K. Entanglement of a pair of quantum bits. Phys. Rev. Lett. 1997, 78, 5022-5025.

66. Wootters, W.K. Entanglement of Formation of an Arbitrary State of Two Qubits. Phys. Rev. Lett. 1998 , 80, 2245-2248. 
67. Horodecki, R.; Horodecki, M. Information-theoretic aspects of inseparability of mixed states. Phys. Rev. A 1996, 54, 1838-1843.

68. Nielsen, M.A.; Kempe, M. Separable states are more disordered hlobally than locally. Phys. Rev. Lett. 2001, 86, 5184-5187.

69. Rossignoli, R; Canosa, N. Generalized entropic criterion for separability. Phys. Rev. A 2002, 66, 042306.

70. Rossignoli, R; Canosa, N. Violation of majorization relations in entangled states and its detection by means of generalized entropic forms. Phys. Rev. A 2003, 67, 042302.

71. Wehrl, H. General properties of entropy. Rev. Mod. Phys. 1978, 50, 221-260.

72. Gigena, N.; Rossignoli, R. Generalized conditional entropy optimization for qudit-qubit states. Phys. Rev. A 2014, 90, 042318.

73. Gigena, N.; Rossignoli, R. Generalized conditional entropy in bipartite systems. J. Phys. A 2014, 47, 015302.

74. Vedral, V. The role of relative entropy in quantum information theory. Rev. Mod. Phys. 2002, 74, 197-234.

75. Canosa, N.; Rossignoli, R. Generalized Nonadditive Entropies and Quantum Entanglement. Phys. Rev. Lett. 2002, 88, 170401.

76. Bhatia, R. Matrix Analysis; Springer: New York, NY, USA, 1997.

77. Marshall, A.; Olkin, I. Inequalities: Theory of Majorization and its Applications; Academic Press: New York, NY, USA, 1979.

78. Filip, R. Overlap and entanglement-witness measurements. Phys. Rev. A 2002, 65, 062320.

79. Nakazato, H.; Tanaka, T.; Yuasa, K.; Florio, G.; Pascazio, S. Measurement scheme for purity based on two two-body gates. Phys. Rev. A 2012, 85, 042316.

80. Tanaka, T.; Kimura, G.; Nakazato, H. Possibility of a Minimal Purity-Measurement Scheme Critically depends on the parity of dimension of the quantum system. Phys. Rev. A 2013, 87, 012303.

81. Tsallis, C. Possible generalization of Boltzmann-Gibbs statistics. J. Stat. Phys. 1988, 52, 479-487.

82. Tsallis, C. Introduction to non-Extensive Statistical Mechanics; Springer: New York, NY, USA, 2009.

83. Piani, M. Problem with the geometric discord. Phys. Rev. A 2012, 86, 034101.

84. Vinjanampathy, S.; Rau, A.R.P. Quantum Discord for qubit-qudit systems. J. Phys. A 2012, 45, 095303.

85. Lieb, E.; Schultz, T.; Mattis, D. Two soluble models of an antiferromagnetic chain. Ann. Phys. 1961, 16, 407-466.

86. De Pasquale, A.; Facchi, P. XY model on the circle: Diagonalization, spectrum, and forerunners of the quantum phase transition. Phys. Rev. A 2009, 80, 032102. 
87. Canosa, N.; Rossignoli, R. Entanglement between distant qubits in cyclic $X X$ chains. Phys. Rev. A 2007, 75, 032350 .

(c) 2015 by the authors; licensee MDPI, Basel, Switzerland. This article is an open access article distributed under the terms and conditions of the Creative Commons Attribution license (http://creativecommons.org/licenses/by/4.0/). 